\title{
Hyperfine Coupling Constants in Local Exact Two-Component Theory
}

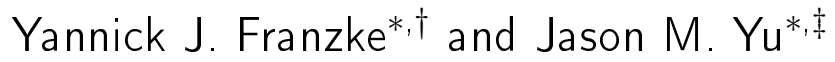 \\ $†$ Fachbereich Chemie, Philipps-Universität Marburg, Hans-Meerwein-Straße 4, 35032 \\ Marburg, Germany \\ $\ddagger$ Department of Chemistry, University of California, Irvine, 1102 Natural Sciences II, \\ Irvine, CA 92697-2025, USA \\ E-mail: yannick.franzke@chemie.uni-marburg.de; yujm1@uci.edu
}

\begin{abstract}
We present a highly efficient implementation of the electron-nucleus hyperfine coupling matrix within one-electron exact two-component (X2C) theory. The complete derivative of the $\mathrm{X} 2 \mathrm{C}$ Hamiltonian is formed, i.e. the derivatives of the unitary decoupling transformation are considered. This requires solution of the response and Sylvester equations, consequently increasing the computational costs. Therefore, we apply the diagonal local approximation to the unitary decoupling transformation (DLU). The finite nucleus model is employed for both the scalar potential and the vector potential. Twoelectron picture-change effects are modeled with the (modified) screened-nuclear spinorbit approach. Our implementation is fully integral direct and OpenMP-parallelized. An extensive benchmark study regarding the Hamiltonian, the basis set, and the density functional approximation is carried out for a set of 12-17 transition-metal compounds. The error introduced by DLU is negligible and the DLU-X2C Hamiltonian accurately reproduces its four-component "fully" relativistic parent results. Functionals with a large amount of Hartree-Fock exchange such as CAM-QTP-02 and $\omega$ B97X-D are generally
\end{abstract}


favorable. The pure density functional $\mathrm{r}^{2} \mathrm{SCAN}$ performs remarkably and even outperforms the common hybrid functionals TPSSh and CAM-B3LYP. Fully uncontracted basis sets or contracted quadruple- $\zeta$ bases are required for accurate results. The capability of our implementation is demonstrated for $\left[\mathrm{Pt}\left(\mathrm{C}_{6} \mathrm{Cl}_{5}\right)_{4}\right]^{-}$with more than 4700 primitive basis functions and four rare-earth single molecule magnets: $\left[\mathrm{La}\left(\mathrm{OAr}^{*}\right)_{3}\right]^{1-}$, $\left[\mathrm{Lu}\left(\mathrm{NR}_{2}\right)_{3}\right]^{1-},\left[\mathrm{Lu}\left(\mathrm{OAr}^{*}\right)_{3}\right]^{1-}$, and $\left[\mathrm{TbPc}_{2}\right]^{-}$. Here, the spin-orbit DLU-X2C Hamiltonian results in an excellent agreement with the experimental findings of all Pt, La, Lu, and $\mathrm{Tb}$ molecules.

\section{Introduction}

The accurate description of electron-nucleus hyperfine interactions in chemical systems is essential for the interpretation of electron paramagnetic resonance (EPR) spectra and the in silico design of novel magnetic materials. First principles prediction of hyperfine spectra, for example, can be used to facilitate the discovery of molecular qubits which exhibit socalled "clock transitions", a type of hyperfine transition that is particularly resilient against quantum decoherence, ${ }^{112}$ for quantum computing applications. ${ }^{[3}$ Realizing methods which efficiently and accurately calculate the hyperfine structure of high-interest systems and guide synthetic efforts is thus a prominent goal of the molecular magnetism community.

A variety of computational approaches are routinely employed to approximate the hyperfine structure of molecules such as the complete or restricted active space self-consistent field (CASSCF/RASSCF), ${ }^{415}$ density functional theory (DFT), ${ }^{6}$ and correlated wavefunction methods. ${ }^{788}$ Due to the known sensitivity of hyperfine interactions to relativistic effects, $\frac{9}{9}$ scalar-relativistic corrections ${ }^{10}$ are also commonly included to different levels of approximate two-component theory, and used with a non-relativistic hyperfine operator. ${ }^{11}$ These methods have been demonstrated to perform well for organic radicals and metal complexes with valence shells of high orbital angular momentum.

However, calculating the hyperfine interaction becomes significantly more complex for 
systems containing heavy elements or nearly degenerate electronic states, where spin-orbit coupling effects become large. ${ }^{11-21}$ For example, scalar-relativistic DFT was found to exhibit large errors in the hyperfine coupling tensor predicted for a series of $\mathrm{La}(\mathrm{II})$ and $\mathrm{Lu}(\mathrm{II})$-based organometallic complexes, likely due to the divergence of the relativistic spin density near the nucleus and subsequent overestimation of the non-relativistic Fermi-contact term, resulting in errors reaching an order of magnitude. ${ }^{22}$ These large errors also presumably occur in CASSCF, which can be inferred from the unexpectedly large hyperfine couplings recently reported for a $\mathrm{Tb}(\mathrm{II})$-based complex with similar electronic structure. ${ }^{23}$ Thus, to study single molecule magnets, which include the prospective molecular qubit systems mentioned previously, the method must accurately treat both scalar-relativistic and spin-orbit coupling effects and also use the correct relativistic hyperfine expression.

Four-component (4c) and two-component (2c) relativistic methods for calculating EPR parameters at the DFT level have been previously explored, with the implementation by Malkin et al. ${ }^{[24}$ in a Douglas-Kroll-Hess (DKH) framework, $\stackrel{25+27}{27}$ the application of the zerothorder regular approximation ${ }^{28+30}$ (ZORA) by Autschbach and co-workers ${ }^{12131}$ to the HFC coupling constant, and the four-component level implementation in the groups of Malkin and Kaupp ${ }^{32133}$ representing major milestones. More recently, EPR parameters calculated via the exact two-component $(\mathrm{X} 2 \mathrm{C})$ Hamiltonian $\frac{34}{40}$ were reported independently by the group of Autschbach ${ }^{4142}$ as well as Wodyński and Kaupp. ${ }^{43}$ X2C represents a robust ansatz with general application to chemical systems. $\frac{1744445}{}$ However, the aforementioned X2C implementations neglect the derivatives of the $\mathrm{X} 2 \mathrm{C}$ renormalization and decoupling matrices when calculating the derivative of the X2C Hamiltonian, $\frac{46 \mid 50}{5 n}$ introducing an additional approximation into describing the hyperfine tensor. Furthermore, the cost associated with these methods precludes their use for large chemical systems or materials. The relativistic decoupling is performed in the fully decontracted or uncontracted basis which consequently results in considerable memory demands and computation times for molecules with several thousand basis functions ${ }^{51}$ due to the $\mathcal{O}\left(N^{3}\right)$ scaling of the diagonalization of the 4c Dirac 
matrix, where $N$ measures the system size.

Here, we introduce a new implementation of the quasi-relativistic X2C approach that includes the full derivative of the spin-orbit X2C Hamiltonian and additionally utilizes the diagonal local approximation to the unitary decoupling transformation ${ }^{45}$ (DLU) to achieve highly efficient calculations of the hyperfine coupling matrix. To begin, we briefly review the expression of the relativistic hyperfine coupling matrix before presenting the calculation of $\mathrm{X} 2 \mathrm{C}$ and DLU-X2C Hamiltonian derivatives as implemented in this work. We then demonstrate our method's accuracy and the speed-up achieved by using DLU through comparison

of the hyperfine matrix with that obtained from the $4 \mathrm{c}$ method of Gohr et al. ${ }^{[33}$ for a series of transition-metal complexes. Efficiency is further demonstrated for a larger platinum complex $\left[\mathrm{Pt}\left(\mathrm{C}_{6} \mathrm{Cl}_{5}\right)_{4}\right]^{-}$described with 4700 primitive basis functions. We additionally evaluate the error introduced from neglecting the decoupling and renormalization matrix derivatives for these systems. It is shown that the error introduced by DLU is smaller than the latter. We go on to conduct an evaluation of commonly used relativistic all-electron basis sets and density functional approximations to identify best practices for general application to molecules. Finally, we apply our method to compute the isotropic hyperfine coupling constants for a series of rare-earth single molecule magnets, demonstrating its novel capacity to describe these systems of great interest.

\section{Theory}

\subsection{Notation}

As done previously for DLU-X2C,,$\sqrt[5155]{55}$ we use the matrix notation of Reiher and Wolf. 20 Matrices in the space of spin-free or one-component (1c) basis functions $\left\{\lambda_{\mu}\right\}$ are indicated as $\mathbf{M}$, while matrices in the basis of two-component (2c) functions $\left\{\phi_{\mu}\right\}$ are written as $\boldsymbol{M}$. The $2 \mathrm{c}$ spinor basis functions are chosen as the direct product of the scalar basis functions with spin functions, $\left\{\lambda_{\mu}\right\} \otimes\{\alpha, \beta\}$. M refers to the corresponding matrices in the space of four- 
component (4c) functions. A split notation for large (L) and small (S) components is used. The subscripts - and + refer to the so-called positronic and the electronic states. Cartesian coordinates are denoted with $u, v$. Furthermore, atomic units are used throughout unless explicitly stated otherwise. Gaussian-based units are used for the magnetic interaction. The corresponding superscripts and subscripts refer to the derivative, i.e.

$$
\boldsymbol{h}_{u}^{N}=\left(\frac{\mathrm{d} \boldsymbol{h}}{\mathrm{d} m_{N, u}}\right)_{m_{N, u}=0}
$$

Note that the derivatives are formed in the limit of a vanishing perturbation.

\subsection{Hyperfine Coupling Constant}

The electron paramagnetic resonance (EPR) spin Hamiltonian ${ }^{13}$ for a given nucleus $N$ reads

$$
\hat{H}_{N}^{\mathrm{EPR}}=\vec{I}_{N} \stackrel{\leftrightarrow}{A}_{N} \vec{S}
$$

The hyperfine coupling (HFC) matrix or so-called hyperfine coupling tensor $\stackrel{\leftrightarrow}{A}_{N}$ is defined as the second derivative of the energy with respect to the electron spin $\vec{S}$ and nuclear spin $\vec{I}$

$$
A_{N, u v}=\frac{\mathrm{d}^{2} E}{\mathrm{~d} I_{N, u} \mathrm{~d} S_{v}}=g_{N} \beta_{N} \frac{\mathrm{d}^{2} E}{\mathrm{~d} m_{N, u} \mathrm{~d} S_{v}}
$$

Here, $g_{N}$ denotes the nuclear $g$-factor, $\beta_{N}$ is the nuclear magneton, and $\vec{m}_{N}$ is the magnetic dipole moment of nucleus $N$. In non-relativistic approaches, the hyperfine coupling is commonly split into the Fermi-contact (FC) and the spin-dipole (SD) interaction, see for instance the discussion in Refs. 7, and 31. The Fermi-contact term only contributes to the isotropic HFC constant and is commonly computed with the excess spin density $\rho^{\alpha-\beta}$ at the origin of the nucleus, $\vec{N}$, according to

$$
A_{N, \text { iso }}^{\mathrm{FC}}=\frac{4 \pi}{3} g_{N} \beta_{N}\left\langle\hat{S}_{z}\right\rangle^{-1} \rho^{\alpha-\beta}(\vec{N})
$$


Note that this expression exploits the point charge model. In contrast, the spin-dipole interaction contributes to the anisotropy

$$
A_{N, u v}^{\mathrm{SD}}=\frac{1}{2} g_{N} \beta_{N}\left\langle\hat{S}_{z}\right\rangle^{-1} \sum_{\mu \nu} \mathbf{P}_{\mu \nu}^{\alpha-\beta}\left\langle\lambda_{\mu}\left|\hat{r}_{N}^{-5}\left[\hat{r}_{N}^{2} \delta_{u v}-3 \hat{r}_{N, u} \hat{r}_{N, v}\right]\right| \lambda_{\nu}\right\rangle
$$

with $\vec{r}_{N}=\vec{r}-\vec{N}$ and the excess spin density matrix $\mathbf{P}_{\mu \nu}^{\alpha-\beta}$. Additionally, $\delta_{u v}$ is the Kronecker delta and $r$ is the shorthand notation for $|\vec{r}|$.

In a quasi-relativistic $2 \mathrm{c}$ framework, the spin derivatives are evaluated using three generalized collinear calculations ${ }^{12 / 41 / 56 \mid 57}$ or the generalized non-collinear approach $2432133 \mid 58$ with the spin aligned along a coordinate axis. Herein, we adopt the latter ansatz as the noncollinear formalism incorporates spin polarization effects and is straightforwardly applicable beyond Kramers doublets. Also, this approach is commonly used in 4c calculations. $\frac{32|33| 58}{36}$ The energy depends on the orientation of the magnetization vector, $\vec{J}=(\vec{S}+\vec{L})$, along the axis $v . \vec{L}$ denotes the angular momentum. The hyperfine coupling matrix reads $s^{24|32| 33}$

$$
A_{N, u v}=\frac{g_{N} \beta_{N}}{\left\langle\tilde{S}_{v}\right\rangle} \frac{\mathrm{d} E\left(J_{v}, \vec{I}_{N}\right)}{\mathrm{d} I_{N, u}}=\frac{g_{N} \beta_{N}}{\left\langle\tilde{S}_{v}\right\rangle} \operatorname{tr}\left[\boldsymbol{h}_{u}^{N} \boldsymbol{P}\left(J_{v}\right)\right]
$$

$\left\langle\tilde{S}_{v}\right\rangle$ is the effective spin along the direction $v$ and $\boldsymbol{P}$ denotes the two-component density matrix. Thus, three SCF calculations with orthogonal magnetization $J_{v}$ are required. The effective spin is formally given by the electronic ground state and its term symbol. In case of symmetry the three axes are already defined. For large or non-symmetric molecules, a proper orientation of the molecule is required to directly obtain the HFC tensor in its diagonal form. ${ }^{24 \mid 32 \sqrt{3} 3}$ To evaluate Eq. 6 the derivative of the $\mathrm{X} 2 \mathrm{C}$ or the DLU-X2C Hamiltonian is required - similar to nuclear magnetic resonance (NMR) coupling constants. $\frac{55 \mid 59}{}$ We will first discuss the (full) $\mathrm{X} 2 \mathrm{C}$ approach and then apply the DLU scheme. Here, we aim for a selfcontained derivation for an implementation-ready formulation. Emphasis will be put on the derivatives of the unitary decoupling transformation. 


\subsection{Derivatives of the X2C Hamiltonian}

The formulation of the complete derivatives in one-electron $\mathrm{X} 2 \mathrm{C}$ requires the following four major steps. First, the Dirac equation is constructed in the presence of a magnetic perturbation. Second, a (finite) basis set is introduced to arrive at its matrix representation. Third, the unitary decoupling step in the spirit of Foldy and Wouthuysen ${ }^{60}$ is carried out. Fourth, the actual derivatives of the $\mathrm{X} 2 \mathrm{C}$ Hamiltonian with respect of the magnetic perturbation are formed using the product rule.

To account for magnetic perturbations, the generalized momentum operator $\hat{\vec{\pi}}$ is introduced according to the principle of minimal coupling

$$
\hat{\vec{\pi}}=\hat{\vec{p}}+\frac{1}{c} \hat{\overrightarrow{\mathcal{A}}}=\hat{\vec{p}}+\frac{1}{c} \sum_{N} \hat{\overrightarrow{\mathcal{A}}}_{N}
$$

where $\hat{\vec{p}}$ is the linear momentum operator and $\overrightarrow{\mathcal{A}}$ is the vector potential associated with the nuclear magnetic moments. The vector potential in a finite nucleus model is given by $\frac{62[63}{6}$

$$
\begin{aligned}
\overrightarrow{\mathcal{A}}_{N}(\vec{r}) & =\vec{m}_{N} \times \vec{\nabla}_{N} G_{N}(\vec{r}) \\
G_{N}(\vec{r}) & =\int \frac{w_{N}\left(\vec{R}_{N}\right)}{|\vec{r}-\vec{R}|} d \vec{R}
\end{aligned}
$$

where $w_{N}$ describes the shape of the nuclear charge distribution. For a Gaussian charge distribution and a nucleus placed at the position $\vec{N}$, the shape function $w_{N}$ reads

$$
\begin{aligned}
w_{N}\left(\vec{R}_{N}\right) & =\left(\frac{\eta}{\pi}\right)^{3 / 2} \exp \left(-\eta R_{N}^{2}\right) \\
\vec{R}_{N} & =\vec{R}-\vec{N}
\end{aligned}
$$

Parameters for the exponent $\eta$ in this model are available in Ref. 64. Replacing the linear momentum $\hat{\vec{p}}$ by its generalization $\hat{\vec{\pi}}$ in the one-electron Dirac-Hamilton operator and 
subsequent expansion in a restricted kinetically balanced (RKB) basis set $\frac{65}{6}$ according to

$$
\begin{aligned}
\left|\psi_{i}^{\mathrm{L}}\right\rangle & =\sum_{\mu} c_{\mu i}^{\mathrm{L}}\left|\phi_{\mu}\right\rangle \\
\left|\psi_{i}^{\mathrm{S}}\right\rangle & =\sum_{\mu} c_{\mu i}^{\mathrm{S}} \frac{\vec{\sigma} \cdot \hat{\vec{p}}}{2 c}\left|\phi_{\mu}\right\rangle
\end{aligned}
$$

leads to the one-electron Dirac equation in a matrix representation

$$
\mathbb{D} \mathbb{C}=\mathbb{M} \mathbb{C E}
$$

In the super-matrix form, this reads

$$
\left(\begin{array}{cc}
\boldsymbol{V} & \boldsymbol{\Pi}^{\dagger} \\
\boldsymbol{\Pi} & \left(\frac{1}{4 c^{2}} \boldsymbol{W}-\boldsymbol{T}\right)
\end{array}\right)\left(\begin{array}{cc}
\boldsymbol{C}_{-}^{\mathrm{L}} & \boldsymbol{C}_{+}^{\mathrm{L}} \\
\boldsymbol{C}_{-}^{\mathrm{S}} & \boldsymbol{C}_{+}^{\mathrm{S}}
\end{array}\right)=\left(\begin{array}{cc}
\boldsymbol{S} & \mathbf{0}_{2} \\
\boldsymbol{0}_{2} & \frac{1}{2 c^{2}} \boldsymbol{T}
\end{array}\right)\left(\begin{array}{cc}
\boldsymbol{C}_{-}^{\mathrm{L}} & \boldsymbol{C}_{+}^{\mathrm{L}} \\
\boldsymbol{C}_{-}^{\mathrm{S}} & \boldsymbol{C}_{+}^{\mathrm{S}}
\end{array}\right)\left(\begin{array}{ll}
\boldsymbol{\epsilon}_{-} & \mathbf{0}_{2} \\
\boldsymbol{0}_{2} & \boldsymbol{\epsilon}_{+}
\end{array}\right)
$$

Here, $\boldsymbol{S}, \boldsymbol{T}$, and $\boldsymbol{V}$ are the overlap matrix, the kinetic energy matrix, and the scalar potential

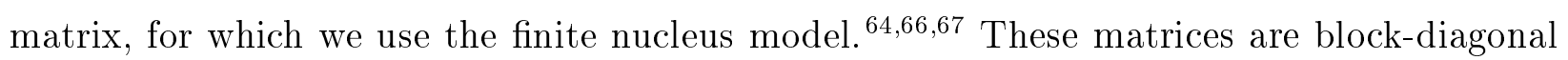
in the two-component space. The generalized momentum matrix $\boldsymbol{\Pi}$ is not block-diagonal and reads

$$
\boldsymbol{\Pi}_{\mu \nu}^{\dagger}=\left\langle\phi_{\mu}\left|c \vec{\sigma} \cdot\left(\hat{\vec{p}}+\frac{1}{c} \hat{\overrightarrow{\mathcal{A}}}\right)\right|\left(\frac{\vec{\sigma} \cdot \hat{\vec{p}}}{2 c}\right) \phi_{\nu}\right\rangle
$$

We note in passing that quadratic terms in $\hat{\overrightarrow{\mathcal{A}}}$ are obtained by an expansion according to the restricted magnetic (RMB) balance condition. ${ }^{5968}$ However, the impact of RMB is of minor importance for the derivatives with respect to the magnetic moments $\frac{4155169-72}{72}$ and the HFC constant is also evaluated with the RKB condition in 4c approaches. $\frac{32[33}{\text { Matters }}$ are different for NMR shifts $\frac{53 \mid 73-76}{76}$ and magnetic circular dichroism. ${ }^{[77}$ The relativistically 
modified potential $\boldsymbol{W}$ can be evaluated from four real matrices $\mathbf{W}^{0}, \mathbf{W}^{x}, \mathbf{W}^{y}$, and $\mathbf{W}^{z}$ as

$$
\boldsymbol{W}_{\mu \nu}=\left(\begin{array}{cc}
\mathbf{W}^{0}+\mathrm{i} \mathbf{W}^{z} & \mathbf{W}^{y}+\mathrm{i} \mathbf{W}^{x} \\
-\mathbf{W}^{y}+\mathrm{i} \mathbf{W}^{x} & \mathbf{W}^{0}-\mathrm{i} \mathbf{W}^{z}
\end{array}\right)
$$

with

$$
\begin{aligned}
\mathbf{W}_{\mu \nu}^{0} & =\left\langle\lambda_{\mu}\left|\hat{p}_{x} \hat{V} \hat{p}_{x}+\hat{p}_{y} \hat{V} \hat{p}_{y}+\hat{p}_{z} \hat{V} \hat{p}_{z}\right| \lambda_{\nu}\right\rangle \\
\mathbf{W}_{\mu \nu}^{x} & =\left\langle\lambda_{\mu}\left|\hat{p}_{y} \hat{V} \hat{p}_{z}-\hat{p}_{z} \hat{V} \hat{p}_{y}\right| \lambda_{\nu}\right\rangle \\
\mathbf{W}_{\mu \nu}^{y} & =\left\langle\lambda_{\mu}\left|\hat{p}_{z} \hat{V} \hat{p}_{x}-\hat{p}_{x} \hat{V} \hat{p}_{z}\right| \lambda_{\nu}\right\rangle \\
\mathbf{W}_{\mu \nu}^{z} & =\left\langle\lambda_{\mu}\left|\hat{p}_{x} \hat{V} \hat{p}_{y}-\hat{p}_{y} \hat{V} \hat{p}_{x}\right| \lambda_{\nu}\right\rangle
\end{aligned}
$$

$\mathbf{W}^{0}$ is a symmetric matrix whereas $\mathbf{W}^{x}, \mathbf{W}^{y}$, and $\mathbf{W}^{z}$ are antisymmetric matrices. Within the (modified) screened nuclear spin-orbit (SNSO, mSNSO) approximation, $\frac{78}{81}$ the integrals for the spin-dependent parts $\left(\mathbf{W}^{x}, \mathbf{W}^{y}, \mathbf{W}^{z}\right)$ are re-scaled to (approximately) account for the missing two-electron picture-change effects. $.80[82$

Application of the unitary decoupling transformation yields the X2C Hamiltonian $\underline{34} 40$

$$
\boldsymbol{h}^{+}=\boldsymbol{R}^{\dagger} \boldsymbol{L} \boldsymbol{R}
$$

with the matrix representation of the normalized elimination of the small component (NESC) operator $^{10 \mid 83}+85$

$$
\boldsymbol{L}=\boldsymbol{V}+\boldsymbol{X}^{\dagger} \boldsymbol{\Pi}+\boldsymbol{\Pi}^{\dagger} \boldsymbol{X}+\boldsymbol{X}^{\dagger}\left(\frac{1}{4 c^{2}} \boldsymbol{W}-\boldsymbol{T}\right) \boldsymbol{X}
$$

The decoupling matrix $\boldsymbol{X}$ is obtained in one shot by diagonalization of the Dirac matrix $\mathbb{D}$ with the metric $\mathbb{M}$ and reads

$$
\boldsymbol{X}=\boldsymbol{C}_{+}^{\mathrm{S}}\left(\boldsymbol{C}_{+}^{\mathrm{L}}\right)^{-1}
$$


The renormalization matrix $\boldsymbol{R}$ follows as 3

$$
\begin{aligned}
\boldsymbol{R} & =\boldsymbol{S}^{-1 / 2}\left(\boldsymbol{S}^{-1 / 2} \tilde{\boldsymbol{S}} \boldsymbol{S}^{-1 / 2}\right)^{-1 / 2} \boldsymbol{S}^{1 / 2} \\
\tilde{\boldsymbol{S}} & =\boldsymbol{S}+\frac{1}{2 c^{2}} \boldsymbol{X}^{\dagger} \boldsymbol{T} \boldsymbol{X}
\end{aligned}
$$

Note that the renormalization matrix is non-Hermitian yet positive definite. ${ }^{86}$

Differentiating the $\mathrm{X} 2 \mathrm{C}$ Hamiltonian in Eq. 22 with respect to the magnetic moments yields

$$
\boldsymbol{h}^{+, N}=\boldsymbol{R}^{\dagger, N} \boldsymbol{L} \boldsymbol{R}+\boldsymbol{R}^{\dagger} \boldsymbol{L}^{N} \boldsymbol{R}+\boldsymbol{R}^{\dagger} \boldsymbol{L} \boldsymbol{R}^{N}
$$

where the derivative of the NESC matrix is obtained in a straightforward manner using the product rule

$$
\begin{aligned}
\boldsymbol{L}^{N}= & \boldsymbol{\Pi}^{\dagger, N} \boldsymbol{X}+\boldsymbol{\Pi}^{\dagger} \boldsymbol{X}^{N}+\boldsymbol{X}^{\dagger, N} \boldsymbol{\Pi}+\boldsymbol{X}^{\dagger} \boldsymbol{\Pi}^{N} \\
+ & \frac{1}{4 c^{2}}\left(\boldsymbol{X}^{\dagger, N} \boldsymbol{W} \boldsymbol{X}+\boldsymbol{X}^{\dagger} \boldsymbol{W} \boldsymbol{X}^{N}\right) \\
& -\boldsymbol{X}^{\dagger, N} \boldsymbol{T} \boldsymbol{X}-\boldsymbol{X}^{\dagger} \boldsymbol{T} \boldsymbol{X}^{N}
\end{aligned}
$$

The derivative of the generalized momentum matrix is given by the respective one-electron integral derivatives 55

$$
\left(\boldsymbol{\Pi}_{\mu \nu}^{\dagger}\right)_{u}^{N}=\frac{1}{2 c}\left\langle\phi_{\mu}\left|\left(\vec{\nabla}_{N} \hat{G}_{N} \times \vec{\sigma}\right)_{u}\right|(\vec{\sigma} \cdot \hat{\vec{p}}) \phi_{\nu}\right\rangle
$$

In the non-relativistic limit and the point charge model $(\eta \rightarrow \infty)$, this integral expression reduces to the well known form of the Fermi-contact, the spin-dipole, and the paramagnetic

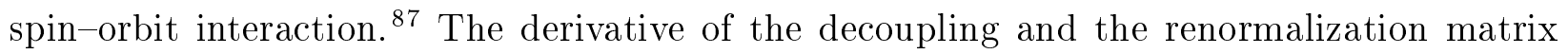
arise for many-electron systems as the decoupling is performed in the presence of a perturbation. $\frac{46[48}{4 s}$ the decoupling matrix depends on the coefficients, response equations are used to form the derivatives. This formalism is similar to coupled-perturbed Hartree-Fock theory, ${ }^{88 / 89}$ however, the response equations are solved in one step for $\mathrm{X} 2 \mathrm{C}$ as only one- 
electron terms are involved. Consequently, the (one-electron) orbital rotation matrix $\mathbb{O}$ is introduced to calculate the so-called perturbed coefficients

$$
\left(\begin{array}{ll}
C_{L-}^{N} & C_{L+}^{N} \\
C_{S-}^{N} & C_{S+}^{N}
\end{array}\right)=\left(\begin{array}{ll}
C_{L-} & C_{L+} \\
C_{S-} & C_{S+}
\end{array}\right)\left(\begin{array}{ll}
O_{--}^{N} & O_{-+}^{N} \\
O_{+-}^{N} & O_{++}^{N}
\end{array}\right)
$$

The elements of the orbital rotation matrix $\boldsymbol{O}_{-+}$can be calculated as

$$
\left(\boldsymbol{O}_{-+}^{N}\right)_{k l}=\frac{\left(\tilde{\boldsymbol{D}}_{-+}^{N}\right)_{k l}}{\left(\boldsymbol{E}_{++}\right)_{l l}-\left(\boldsymbol{E}_{--}\right)_{k k}}
$$

where the notation $\tilde{\boldsymbol{D}}_{-+}^{N}$ indicates that the perturbed Dirac matrix is formed in the basis of the unperturbed solutions according to

$$
\tilde{\boldsymbol{D}}_{-+}^{N}=\boldsymbol{C}_{-}^{L, \dagger} \boldsymbol{\Pi}^{\dagger, N} \boldsymbol{C}_{+}^{S}+\boldsymbol{C}_{-}^{S, \dagger} \boldsymbol{\Pi}^{N} \boldsymbol{C}_{+}^{L}
$$

The numerical stability of Eq. 31 is ensured by the large energetic gap between the so-called positronic and the electronic states. Using the normalization of the large component

$$
C_{L+}^{\dagger} \tilde{S} C_{L+}=\boldsymbol{I}
$$

where $\boldsymbol{I}$ is the unit matrix, the derivative of the decoupling matrix follows as ${ }^{48 \mid 86}$

$$
\boldsymbol{X}^{N}=\left(\boldsymbol{C}_{S_{-}}-\boldsymbol{X} \boldsymbol{C}_{L_{-}}\right) \boldsymbol{O}_{-+}^{N} \boldsymbol{C}_{L+}^{\dagger} \tilde{\boldsymbol{S}}
$$

Therefore, the derivative of the decoupling matrix can be obtained by using simple basic linear algebra subroutines (BLAS). ${ }^{90 \mid 91}$ We refer to the appendix of Ref. 53 for further details on the derivation and a comparison of the different approaches for the one-electron response equations. Finally, the derivative of the renormalization matrix is evaluated based 
on 10

$$
\boldsymbol{R} \boldsymbol{R}=\tilde{\boldsymbol{S}}^{-1} \boldsymbol{S}
$$

This yields the Sylvester matrix equation for the perturbed renormalization matrix

$$
\boldsymbol{R} \boldsymbol{R}^{N}+\boldsymbol{R}^{N} \boldsymbol{R}=\tilde{\boldsymbol{S}}^{-1} \tilde{\boldsymbol{S}}^{N} \boldsymbol{R} \boldsymbol{R}
$$

with the derivative $\tilde{\boldsymbol{S}}^{N}$ of the right-hand side given by

$$
\tilde{\boldsymbol{S}}^{N}=\frac{1}{2 c^{2}} \boldsymbol{X}^{\dagger, N} \boldsymbol{T} \boldsymbol{X}+\frac{1}{2 c^{2}} \boldsymbol{X}^{\dagger} \boldsymbol{T} \boldsymbol{X}^{N}
$$

The Sylvester matrix equation is solved with an eigenvalue decomposition method. $\underline{86}$

\subsection{Derivatives of the DLU-X2C Hamiltonian}

The algebraic operations such as matrix multiplications and diagonalizations for the $\mathrm{X} 2 \mathrm{C}$ Hamiltonian and its derivatives are typically carried out in a large uncontracted basis. Therefore, their computation leads to substantially increased demands when compared to the ground-state energy calculation, $\frac{52}{5 \text { wich }}$ are in and of themselves computationally expensive in the $\mathrm{X} 2 \mathrm{C}$ scheme for a few thousand basis functions. $\frac{51}{5}$ Thus, we apply the DLU scheme to reduce the computational overhead for the inclusion of the derivatives of $\boldsymbol{X}$ and $\boldsymbol{R}$. In the non-orthogonal basis set of the last subsection, the unitary decoupling matrix is approximated according to

$$
\begin{aligned}
\boldsymbol{U}^{\mathrm{LL}} & =\bigoplus_{A} \boldsymbol{U}_{A A}^{\mathrm{LL}}=\bigoplus_{A} \boldsymbol{R}_{A A} \\
\boldsymbol{U}^{\mathrm{SL}} & =\bigoplus_{A} \boldsymbol{U}_{A A}^{\mathrm{SL}}=\bigoplus_{A} \boldsymbol{X}_{A A} \boldsymbol{R}_{A A}
\end{aligned}
$$

Here, the atomic blocks are defined according to the atom center of the basis functions for the respective matrix element of the one-electron integrals. We denote an atomic diagonal block 
as $A A$ and a general atomic block as $A B$. Therefore, the atomic blocks of the Hamiltonian are generally defined as

$$
\boldsymbol{h}_{A B}^{+}=\boldsymbol{R}_{A A}^{\dagger} \boldsymbol{L}_{A B} \boldsymbol{R}_{B B}^{\dagger}
$$

which yields the working equation for the ground-state energy

$$
\begin{aligned}
\boldsymbol{h}_{A B}^{+}= & \boldsymbol{R}_{A A}^{\dagger}\left(\boldsymbol{V}_{A B}+\boldsymbol{\Pi}_{A B}^{\dagger} \boldsymbol{X}_{B B}+\boldsymbol{X}_{A A}^{\dagger} \boldsymbol{\Pi}_{A B}\right) \boldsymbol{R}_{B B} \\
& +\boldsymbol{R}_{A A}^{\dagger}\left(\boldsymbol{X}_{A A}^{\dagger}\left[\frac{1}{4 c^{2}} \boldsymbol{W}_{A B}-\boldsymbol{T}_{A B}\right] \boldsymbol{X}_{B B}\right) \boldsymbol{R}_{B B}
\end{aligned}
$$

Accordingly, only the atomic diagonal block of the Dirac matrix is diagonalized to obtain $\boldsymbol{X}_{A A}$ and $\boldsymbol{R}_{A A}$. Therefore, the formal scaling is reduced from $\mathcal{O}\left(N^{3}\right)$ for the full X2C Hamiltonian to $\mathcal{O}(N)$ for the atomic diagonal blocks and overall to $\mathcal{O}\left(N^{2}\right)$ due to the number of atom pairs for the atomic diagonal block. $N$ measures the size of the system.

The derivatives of the DLU-X2C Hamiltonian for the HFC matrix follow as

$$
\begin{aligned}
\boldsymbol{h}_{A B}^{+, N}= & \boldsymbol{R}_{A A}^{\dagger, N} \boldsymbol{L}_{A B} \boldsymbol{R}_{B B}^{\dagger}+\boldsymbol{R}_{A A}^{\dagger} \boldsymbol{L}_{A B} \boldsymbol{R}_{B B}^{\dagger, N} \\
& +\boldsymbol{R}_{A A}^{\dagger}\left(\boldsymbol{\Pi}_{A B}^{\dagger, N} \boldsymbol{X}_{B B}+\boldsymbol{X}_{A A}^{\dagger} \boldsymbol{\Pi}_{A B}^{N}\right) \boldsymbol{R}_{B B} \\
& +\boldsymbol{R}_{A A}^{\dagger}\left(\boldsymbol{\Pi}_{A B}^{\dagger} \boldsymbol{X}_{B B}^{N}+\boldsymbol{X}_{A A}^{\dagger, N} \boldsymbol{\Pi}_{A B}\right) \boldsymbol{R}_{B B} \\
& +\boldsymbol{R}_{A A}^{\dagger}\left(\boldsymbol{X}_{A A}^{\dagger, N}\left[\frac{1}{4 c^{2}} \boldsymbol{W}_{A B}-\boldsymbol{T}_{A B}\right] \boldsymbol{X}_{B B}\right) \boldsymbol{R}_{B B} \\
& +\boldsymbol{R}_{A A}^{\dagger}\left(\boldsymbol{X}_{A A}^{\dagger}\left[\frac{1}{4 c^{2}} \boldsymbol{W}_{A B}-\boldsymbol{T}_{A B}\right] \boldsymbol{X}_{B B}^{N}\right) \boldsymbol{R}_{B B}
\end{aligned}
$$

To evaluate these derivatives, first the atomic diagonal blocks of the unperturbed decoupling matrix $\boldsymbol{X}_{A A}$ and the unperturbed renormalization matrix $\boldsymbol{R}_{A A}$ are computed. Second, the atomic diagonal blocks of the perturbed Hamiltonian are calculated by a straightforward application of the response and Sylvester matrix, Eqs. 30 37. The atomic diagonal blocks $\boldsymbol{X}_{A A}^{N}$ and $\boldsymbol{R}_{A A}^{N}$ are then used to perform the matrix multiplications in Eq. 42 . Note that both the atomic diagonal and atomic off-diagonal blocks of the perturbed generalized momentum matrix $\boldsymbol{\Pi}^{N}$ need to be evaluated. However, the one-electron integrals and integral derivatives 
themselves are negligible in terms of computational demands.

\section{Implementation}

We have implemented the DLU-X2C and X2C approach for HFC constants into the TURBOMOLE $^{92}$ quantum chemistry program suite. The derivatives of the decoupling and the renormalization matrix are obtained based on the one-electron response routines of Refs. 52, 53, and 55. The integral derivatives are taken from the latter references, whereas the parent (unperturbed) integrals were implemented in Ref. 52. Consequently, all integrals are evaluated using Gauss-Rys $\frac{96 \mid 97}{9}$ and Gauss-Hermite integration. Parameters for the Gaussian charge distribution are taken from Ref. 64 and the (modified) screened nuclear spin-orbit approximation is available to re-scale the spin-dependent contributions of $\boldsymbol{W} .78$ Nuclear $g$ factors are calculated based on the available gyromagnetic ratios. ${ }^{98} 100$ A value of $c=137.0359990840$ a.u. ${ }^{101}$ is used for the speed of light in atomic units.

Our implementation in the ridft module $\sqrt[51|52| 102+106]{1-1 s}$ completely integral direct and makes use of the OpenMP parallelization ${ }^{107}$ throughout all integrals and algebraic operations. 108 The Intel ${ }^{\circledR}$ Math Kernel Library (Intel MKL) is further used in this work. The resolution of the identity approximation to the Coulomb integrals, RI- $J, 102 / 109 / 110$ and its multipoleaccelerated variant MARI- $J^{\sqrt{111}}$ as well as to the exchange integrals, RI- $K, \sqrt{102 \mid 112}$ and the seminumerical exchange approximation $\sqrt{104 \mid 105}$ are available. Furthermore, the integrals can be calculated without these approximations. ${ }^{[103[106}$ The conductor-like screening model $\mathrm{l}^{113[114}$ (COSMO) is supported to simulate the counter ions in case of charged systems or to consider the environment in solution. We note that two-component generalized Kohn-Sham calculations require tailored grids for all-electron approaches and therefore we use the grids

of Ref. 115. Currently, functionals up to the fourth rung of Jacob's ladder, $\frac{116-118}{1 n c l u d-}$ ing generalized gradient approximations (GGAs), meta-generalized gradient approximations (meta-GGAs or mGGAs), and their respective (range-separated) hybrids, are supported. In- 
terfaces to Libxc $\frac{119}{121}$ and XCFun $\frac{122}{122}$ are further provided. We validated the implementation by comparison to the scalar-relativistic approach $\frac{123}{123}$ and the non-relativistic limit. 1001123

Pre-processing and post-processing scripts are available so that only converged unrestricted Hartree-Fock or Kohn-Sham (UHF/UKS) orbitals are required and the complete two-component procedure can be carried out in a "black-box" fashion. The spin contributions to the HFC matrix are evaluated at the end of each SCF calculation. The post-processing script assembles the full HFC matrix and calculates the principal components. These may be obtained with the rank-2 tensor $\stackrel{\leftrightarrow}{A}_{N} \stackrel{\leftrightarrow}{A}_{N}^{T}$. The eigenvectors of this quantity form the principal axis system of the HFC and the square root of the eigenvalues are the principal HFC values, i.e. $A_{N, 11}^{\mathrm{PAS}}, A_{N, 22}^{\mathrm{PAS}}$, and $A_{N, 33}^{\mathrm{PAS}}$. Note that the sign information is lost in the rank-2 tensor, and so the signs are determined by comparison to the one-component ansatz and by algebraic considerations, i.e. the sign of the determinant of $\stackrel{\leftrightarrow}{A}_{N}$ and $\stackrel{\leftrightarrow}{A}_{N}^{\text {PAS }}$ is identical. ${ }^{12}$ Another way to transform the HFC matrix to a diagonal form is to form the symmetric contribution, $\frac{1}{2}\left(\overleftrightarrow{A}_{N}+\overleftrightarrow{A}_{N}^{T}\right)$, and diagonalize it. Similar to the 4c implementation in ReSpect, 124 our im-

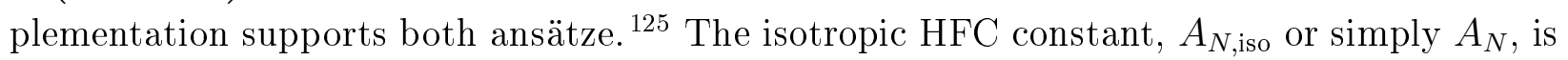
a third of the trace, $A_{N}=\frac{1}{3} \sum_{u} A_{N, u u}^{\mathrm{PAS}}$.

\section{Computational Details}

To begin, we consider the general parameters of a relativistic calculation for a set of transitionmetal compounds in Sec. 5, i.e. the Hamiltonian, the basis set, and the method to treat electron correlation. For the latter, we use DFT. Finally, applications to large molecular systems are presented in Secs. 6 and 7. Note that the finite nucleus model is used for the scalar potential ${ }^{52}$ and the vector potential ${ }^{[5355}$ throughout the main text. MO and spinor files use the ASCII format (American Standard Code for Information Interchange). In the Supporting Information, we further compare our approach to the generalized collinear ZORA ansatz of Verma and Autschbach. 12 
First, the quasi-relativistic two-component X2C and DLU-X2C Hamiltonian are compared to the "fully" relativistic four-component Hamiltonian. This is done for the test set of 17 transition-metal complexes compiled in Ref. [33, namely $\left[\mathrm{MoNCl}_{4}\right]^{2-},\left[\mathrm{MoOF}_{4}\right]^{-}$, $\left[\mathrm{MoOCl}_{4}\right]^{-},\left[\mathrm{MoOF}_{5}\right]^{2-},\left[\mathrm{MoOBr}_{4}\right]^{2-},\left[\mathrm{WOCl}_{4}\right]^{-},\left[\mathrm{WOF}_{5}\right]^{2-},\left[\mathrm{WOBr}_{5}\right]^{2-},\left[\mathrm{TcNF}_{4}\right]^{-},\left[\mathrm{TcNCl}_{4}\right]^{-}$, $\left[\mathrm{TcNBr}_{4}\right]^{-},\left[\mathrm{ReNF}_{4}\right]^{-},\left[\mathrm{ReNCl}_{4}\right]^{-},\left[\mathrm{ReNBr}_{4}\right]^{-},\left[\mathrm{ReOBr}_{4}\right],\left[\mathrm{ReOF}_{5}\right]^{-}$, and $\left[\mathrm{OsOF}_{5}\right]$. Structures are taken from this reference. The same basis sets as therein are employed. Therefore, the uncontracted IGLO-III bases are used for N, F, and $\mathrm{Cl}\left[\frac{126}{12}\right.$ while the Dyall-VTZ basis set is used for $\mathrm{Br}^{\frac{127 / 128}{12}}$ and the Dyall-TZ bases are selected for Mo, Tc, W, Re, and Os. $\frac{128-130}{130}$ Herein, the HFC is calculated with the mSNSO-DLU-X2C and the mSNSO-X2C Hamiltonian in a finite nucleus model. We note that all approaches employ the finite nucleus model for both the scalar and the vector potential. The PBE0 ${ }^{131 \mid 132}$ functional (grid 5a, $115 / 133 \mid 134$ SCF convergence threshold $10^{-9} \mathrm{E}_{\mathrm{h}}$ ) is chosen. While it was shown that an increased amount

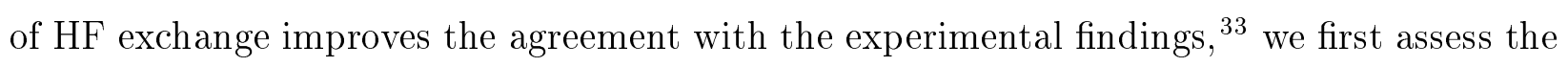
error introduced by DLU and compare the X2C Hamiltonian to its 4c parent. Therefore, the conventional PBE0 functional is chosen for simplicity. The impact of density functional approximations are considered in detail below. Additionally, scalar-relativistic calculations ${ }^{123}$ are carried out. We use the following nuclear $g$ factors: $-0.3653890\left({ }^{95} \mathrm{Mo}\right), 0.2355696\left({ }^{183} \mathrm{~W}\right)$, $1.2632019\left({ }^{43} \mathrm{Tc}\right), 1.2878813\left({ }^{187} \mathrm{Re}\right)$, and $0.4399555\left({ }^{189} \mathrm{Os}\right)$.

Second, we complement the study presented in Ref. 33 at the 4c level with our own basis set study at the $2 \mathrm{c}$ level. The basis sets explored are given in Tab. 1. Note that out of those listed, only the IGLO and the Dyall basis sets overlap in Ref. 33. We chose the large even-tempered (ET) basis set of Ref. 144 as a reference. This basis sets employs 26085 basis functions (spherical AO representation) in total throughout the 17 molecules. The choice of such a large ET basis set in place of experimental findings allows us to consider the impact of the basis set without side effects. All basis sets except for the Dyall and the x2c-type bases are taken from the Basis Set Exchange Library. ${ }^{[148[149}$ Among the chosen segmented-contracted relativistic basis sets, only the Sapporo bases are optimized 
Table 1: Considered basis sets and basis set combinations for the light $(\mathrm{N}, \mathrm{O}, \mathrm{F}, \mathrm{Cl})$ and heavy elements (Br, Mo, Tc, W, Re, Os). The suffix "-unc" denotes that the bases are used in the decontracted form. Note that the ccJ basis sets are only available up to Ne and thus combined with the cc bases. NBF is the total number of spherical AO basis functions. The even-tempered reference basis set uses 26085 basis functions for the molecular test set.

\begin{tabular}{|c|c|c|c|c|c|}
\hline Basis Set & Light Elements & Ref. & Heavy Elements & Ref. & NBF \\
\hline 1 & IGLO-II-unc & 126 & Dyall-VDZ-unc & $127-130$ & 6421 \\
\hline 2 & IGLO-III-unc & 126 & Dyall-VTZ-unc & $127-130$ & 9176 \\
\hline 3 & pcJ-1-unc & 135 & Dyall-VDZ-unc & $127-130$ & 6895 \\
\hline 4 & pcJ-2-unc & 135 & Dyall-VTZ-unc & $127-130$ & 10108 \\
\hline 5 & pcJ-3-unc & 135 & Dyall-VQZ-unc & $127-130$ & 12614 \\
\hline 6 & cc-pVDZ-unc & 136,137 & Dyall-VDZ-unc & $127-130$ & 6440 \\
\hline 7 & cc-pVTZ-unc & 136,137 & Dyall-VTZ-unc & $127-130$ & 9425 \\
\hline 8 & cc-pVQZ-unc & 136137 & Dyall-VQZ-unc & $127-130$ & 11634 \\
\hline 9 & ccJ-pVDZ-unc/cc-pVDZ-unc & $136-138$ & Dyall-VDZ-unc & $127-130$ & 6740 \\
\hline 10 & ccJ-pVTZ-unc/cc-pVTZ-unc & $136-138$ & Dyall-VTZ-unc & $127-130$ & 9614 \\
\hline 11 & ccJ-pVQZ-unc/cc-pVQZ-unc & $136-138$ & Dyall-VQZ-unc & $127-130$ & 11644 \\
\hline 12 & Sapporo-DZP-2012 & 139 & Sapporo-DKH3-DZP-2012 & 139140 & 3369 \\
\hline 13 & Sapporo-TZP-2012 & 139 & Sapporo-DKH3-TZP-2012 & 139140 & 6213 \\
\hline 14 & Sapporo-QZP-2012 & 139 & Sapporo-DKH3-QZP-2012 & 139140 & 9804 \\
\hline 15 & Jorge-DZP-DKH & 141 & Jorge-DZP-DKH & $141-143$ & 3085 \\
\hline 16 & Jorge-TZP-DKH & 141 & Jorge-TZP-DKH & $141-143$ & 5038 \\
\hline 17 & $\mathrm{x} 2 \mathrm{c}-\mathrm{SVPall}-2 \mathrm{c}$ & 106 & $\mathrm{x} 2 \mathrm{c}-\mathrm{SVPall}-2 \mathrm{c}$ & 106 & 3315 \\
\hline 18 & x2c-TZVPall-2c & 106 & x2c-TZVPall-2c & 106 & 5095 \\
\hline 19 & x2c-QZVPall-2c & 144 & x2c-QZVPall-2c & 144 & 8799 \\
\hline 20 & x2c-SVPall-2c-unc & 106 & x2c-SVPall-2c-unc & 106 & 7614 \\
\hline 21 & x2c-TZVPall-2c-unc & 106 & x2c-TZVPall-2c-unc & 106 & 9104 \\
\hline 22 & x2c-QZVPall-2c-unc & 144 & x2c-QZVPall-2c-unc & 144 & 14366 \\
\hline 23 & ANO-R-unc & 145 & ANO-R-unc & 145 & 15764 \\
\hline 24 & ANO-RCC-unc & 146 & ANO-RCC-unc & 146,147 & 15757 \\
\hline
\end{tabular}

with the point charge model. Furthermore, the contractions of the Sapporo and Jorge basis are optimized with the third-order Douglas-Kroll-Hess ${ }^{25}$ (DKH3) Hamiltonian. We note that while the ANO-R basis is optimized for $\mathrm{X} 2 \mathrm{C}$, we employ it in a decontracted form for consistency with the ANO-RCC basis, which is also commonly used in the decontracted form (see for instance Refs. 41,46,74,150,151). As the cc, ccJ, IGLO, and pcJ basis sets are optimized in non-relativistic calculations, they are employed in their decontracted form. Generally, relativistic all-electron Hamiltonians require re-optimized contraction coefficients even for the light elements. $\frac{106|115| 144}{14}$ The $\mathrm{x} 2 \mathrm{c}$-type basis sets are used in both their original 
segmented-contracted fashion and in a fully decontracted fashion. For the latter, we removed functions to avoid linear dependencies and also excluded (nearly) identical functions from the 2c extensions. These basis sets are available in separate files as part of the Supporting Information. We stress that this decontraction is only done to assess the flexibility of these bases, as decontracting segmented-contracted basis sets may easily lead to linear dependencies and convergence issues. Calculations are performed for the transition-metal complexes with the previous settings, i.e. the mSNSO-DLU-X2C Hamiltonian in a finite nucleus model is employed. The thresholds are the same as for the Hamiltonian study and again the PBE0 functional is considered. The mean absolute percent-wise deviation (MAPD) and its standard deviation (STD) are chosen as indicators for the quality of the basis set according to

$$
\operatorname{MAPD}=\sum_{i=1}^{N=17} \frac{\left|A_{i, \text { iso }}^{\text {test }}-A_{i, \text { iso }}^{\text {ref }}\right|}{\left|A_{i, \text { iso }}^{\text {ref }}\right|}
$$

where $A_{\text {iso }}^{\text {test }}$ and $A_{\text {iso }}^{\text {ref }}$ are the isotropic HFC constant for a given basis set and the reference.

Third, the performance of frequently used density functional approximations is studied for 12 of the 17 complexes considered previously. Here, we consider the S-VWN, $152+154$

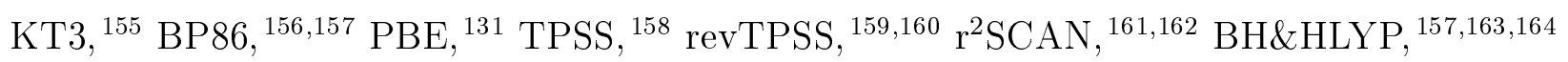

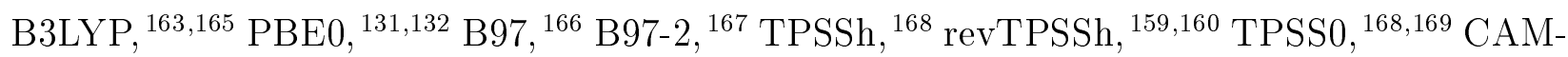

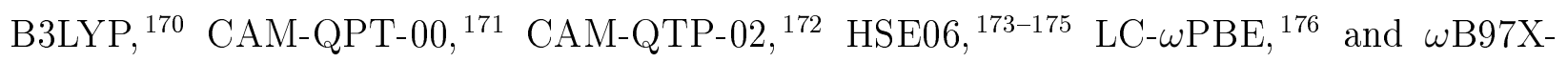
$\mathrm{D}^{177}$ functionals. Libxc $\frac{119}{121}$ is used for the range-separated hybrid functionals, KT3, revTPSS(h), TPSS0, $\mathrm{r}^{2} \mathrm{SCAN}$, and B97 throughout this work. We also use the PBE0 functional including $40 \%$ of $\mathrm{HF}$ exchange (PBE0-40HF) with XCFun. ${ }^{122}$ A review of the construction of each functional is outside the scope of this work, and readers are encouraged to consult the above references for additional details on any DFA used in this study. The $\mathrm{x} 2 \mathrm{c}-\mathrm{QVZP}$ all-2c basis set ${ }^{144}$ is selected and COSMO ${ }^{113[114}$ is applied using the default parameters $^{178}$ to compensate the negative charge. This ensures negative eigenvalues for occupied molecular orbitals and spinors. Having assessed the error of the basis set and the Hamiltonian, the experimental findings $\frac{179-190}{-19}$ collected in Ref. 33 serve as reference. 
In Sec. 6, the impact of the density functional approximation is also studied for a typical application with the mSNSO-DLU-X2C Hamiltonian. Here, the Pt complex $\left[\mathrm{Pt}\left(\mathrm{C}_{6} \mathrm{Cl}_{5}\right)_{4}\right]^{-}$ is considered with the uncontracted Dyall-VTZ basis set ${ }^{191}$ for Pt and the decontracted pcJ-2 bases for $\mathrm{C}$ and $\mathrm{Cl}, \frac{135}{2}$ as well as the segmented-contracted $\mathrm{x} 2 \mathrm{c}-\mathrm{QZVPall}-2 \mathrm{c}$ basis set for all atoms. $\frac{144}{14}$ COSMO is again $\frac{113[114}{10 p p l i e d ~ w i t h ~ t h e ~ d e f a u l t ~ s e t t i n g s ~} \frac{178}{17}$ to compensate the negative charge. The structure is taken from Ref. 33. An SCF threshold of $10^{-8} \mathrm{E}_{\mathrm{h}}$ is applied and large grids are used for the numerical integration of the $\mathrm{XC}$ parts (grid 4a). $\frac{115|133| 134}{}$ Here, the following functionals are chosen. KT3, $\frac{155]}{15 P 86, \frac{156 \mid 157}{, 6}}$

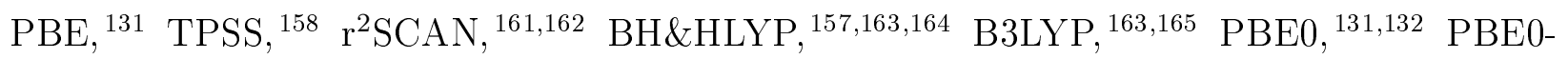

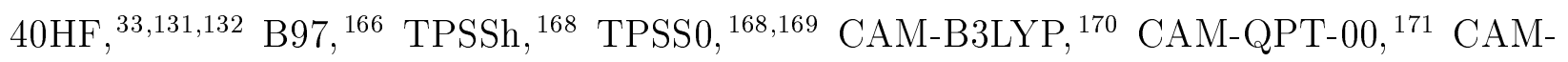

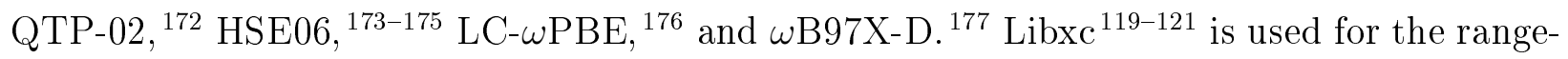
separated hybrid functionals, TPSS0, B97, KT3, and $\mathrm{r}^{2}$ SCAN. Furthermore, calculations with the PBE0-40HF functional are carried out using XCFun. ${ }^{122}$ The nuclear $g$ factor is set to $1.2190420\left({ }^{195} \mathrm{Pt}\right)$. We note that our computational setting with the Dyall-VTZ/pcJ-2 basis leads to 3284 basis functions in the spherical AO representation, whereas the calculations in Ref. 43 employed only 2205 basis functions (Dyall-TZ/IGLO-III). Moreover, a significantly smaller grid was employed in this reference, see Refs. 115, 133, and 134 for the respective integration grids. The x2c-QZVPall-2c basis sets leads to 3003 contracted functions and 4720 primitive functions.

In Sec. 7, we apply the DLU-X2C method to study a series of Ln-based single molecule magnets reported in Ref. 22. The structures given in Ref. 22 were used for all calculations, which were themselves optimized using DFT with the TPSS functional to a Cartesian gradient norm of $10^{-4}$ atomic units and confirmed to be minima via vibrational analysis. Tailored integration grids (grid 4a) are used. $\frac{[15]}{}$ COSMO $\frac{113[114}{}$ was employed to model solvation effects using an epsilon value $\left(\epsilon_{r}\right)$ of 7.520 , solvation radius of $1.30 \AA$, and refractive index of 1.4050 (tetrahydrofuran, THF). D3 dispersion corrections ${ }^{[192}$ were also used. SCF thresholds of $10^{-7} \mathrm{E}_{\mathrm{h}}$ for the energy and $10^{-7}$ a.u. for the root mean square of the density matrix indicate 
the convergence. This methodology to characterize the ground-state electronic structure has been extensively used and validated in previous studies from our group on lanthanide and actinide complexes. $\frac{193}{198}$ The $2 \mathrm{c}$ studies herein include the mSNSO approximation. Details regarding the basis sets and functionals explored for the DLU-X2C calculations, as well as comparisons with the computational methodology used to approximate the HFC in Ref. 22 are discussed in Sec. 7. The nuclear $g$ factors are $0.7951560\left({ }^{139} \mathrm{La}\right)$ and $0.6379065\left({ }^{175} \mathrm{Lu}\right)$.

Finally, we apply the developed methodology to systems with more than one unpaired electron. In Sec.8, we study the compound $\left[\mathrm{TbPc}_{2}\right]^{-}$, where Pc denotes bis(phthalocyaninato), with six unpaired electrons $(S=3)$ using the same computational settings as in Sec. 7. Note that fractional occupation numbers $\frac{199}{199}(\mathrm{FON})$ are used to accelerate the SCF convergence with the $\mathrm{r}^{2} \mathrm{SCAN}$ functional. We employ the same structure as in Ref. 5, which was itself determined in Ref. 200 . The nuclear $g$ factor is 1.3427523 for ${ }^{159} \mathrm{~Tb}$.

\section{Assessment of Accuracy}

\subsection{Comparison to Four-Component Results}

The scalar-relativistic and the spin-orbit X2C Hamiltonians are compared to the parent fourcomponent Dirac-Kohn-Sham (DKS) ansatz in Tab. 2 for 17 transition-metal compounds. Overall, the quasi-relativistic X2C and the DLU-X2C Hamiltonian sufficiently reproduce the DKS results. The DLU error is negligible and typically amounts to about $0.1 \mathrm{MHz}$. In comparison, the impact of spin-orbit coupling amounts to 10-200 MHz. As expected, spinorbit effects are less pronounced for the $4 \mathrm{~d}$ elements than for the $5 \mathrm{~d}$ elements. The Fermicontact term is the leading contribution for these molecules featuring a doublet ground-state configuration. The derivatives are of minor importance for the $4 \mathrm{~d}$ elements (less than $1 \mathrm{MHz}$ ) but their impact rises for the 5d elements. For instance, the inclusion of the derivatives of $\boldsymbol{X}$ and $\boldsymbol{R}$ changes the HFC constant by about $2-3 \mathrm{MHz}$ for the Re compounds. For this assessment, the derivatives of $\boldsymbol{X}$ and $\boldsymbol{R}$ were set to zero in the respective response equations. 
Table 2: Principal components of the hyperfine coupling constant in MHz. Non-collinear 4c results are taken from the Supporting Information of Ref. 33. "PCC" denotes that the derivatives of $\boldsymbol{X}$ and $\boldsymbol{R}$ are neglected, whereas SR and SO refer to scalar-relativistic and spin-orbit calculations. Experimental results $\frac{179}{190}$ were collected in Ref. 33.

\begin{tabular}{|c|c|c|c|c|c|c|c|c|c|}
\hline Molecule & Hamiltonian & $A_{\text {iso }}$ & $A_{\|}$ & $A_{\perp}$ & Molecule & Hamiltonian & $A_{\text {iso }}$ & $A_{\|}$ & $A_{\perp}$ \\
\hline \multirow[t]{7}{*}[\mathrm{MoNCl}_{4}]{$^{2-}$} & $\mathrm{SR} \mathrm{X} 2 \mathrm{C}$ & 162.0 & 227.6 & 129.1 & \multirow[t]{7}{*}[\mathrm{TcNF}_{4}]{$^{-}$} & $\mathrm{SR} \mathrm{X} 2 \mathrm{C}$ & -589.3 & -884.9 & -441.5 \\
\hline & SR DLU & 162.1 & 227.6 & 129.1 & & SR DLU & -589.3 & -884.9 & -441.5 \\
\hline & $\mathrm{SO} \times 2 \mathrm{C} \mathrm{PCC}$ & 175.2 & 260.6 & 132.5 & & $\mathrm{SO} \times 2 \mathrm{C} \mathrm{PCC}$ & -647.9 & -1024.6 & -459.6 \\
\hline & $\mathrm{SO} \times 2 \mathrm{C}$ & 175.3 & 260.7 & 132.6 & & $\mathrm{SO} \mathrm{X} 2 \mathrm{C}$ & -648.5 & -1025.3 & -460.2 \\
\hline & SO DLU & 175.3 & 260.7 & 132.6 & & SO DLU & -648.6 & -1025.4 & -460.1 \\
\hline & $4 \mathrm{c}$ & 176 & 262 & 133 & & $4 c$ & -650 & -1031 & -460 \\
\hline & Expt. & - & - & - & & Expt. & -734 & -1129 & -537 \\
\hline \multirow{7}{*}[\mathrm{MoOF}_{4}]{$^{-}$} & $\mathrm{SR} \times 2 \mathrm{C}$ & 139.3 & 216.1 & 100.8 & \multirow[t]{7}{*}[\mathrm{TcNCl}_{4}]{$^{-}$} & $\mathrm{SR} \times 2 \mathrm{C}$ & -463.9 & -727.3 & -332.2 \\
\hline & SR DLU & 139.3 & 216.1 & 100.8 & & SR DLU & -464.0 & -727.3 & -332.3 \\
\hline & $\mathrm{SO} \times 2 \mathrm{C} P C$ & 157.4 & 251.1 & 110.6 & & $\mathrm{SO} \times 2 \mathrm{C} P C$ & -498.1 & -805.1 & -344.6 \\
\hline & $\mathrm{SO} \times 2 \mathrm{C}$ & 157.6 & 251.3 & 110.7 & & $\mathrm{SO} \times 2 \mathrm{C}$ & -498.6 & -805.8 & -345.1 \\
\hline & SO DLU & 157.6 & 251.3 & 110.7 & & SO DLU & -498.6 & -805.7 & -345.0 \\
\hline & $4 \mathrm{c}$ & 158 & 253 & 111 & & $4 c$ & -500 & -810 & -345 \\
\hline & Expt. & - & 268 & - & & Expt. & -561 & -878 & -402 \\
\hline \multirow[t]{7}{*}[\mathrm{MoOCl}_{4}]{$^{-}$} & $\mathrm{SR} X 2 \mathrm{C}$ & 109.1 & 177.8 & 74.8 & \multirow[t]{7}{*}[\mathrm{TcNBr}_{4}]{$^{-}$} & $\mathrm{SR} X 2 \mathrm{C}$ & -437.4 & -696.7 & -307.8 \\
\hline & SR DLU & 109.1 & 177.8 & 74.8 & & SR DLU & -437.5 & -696.7 & -307.8 \\
\hline & $\mathrm{SO} \times 2 \mathrm{C} P C$ & 122.6 & 201.9 & 82.9 & & $\mathrm{SO} \times 2 \mathrm{C} P C$ & -432.8 & -668.8 & -314.8 \\
\hline & $\mathrm{SO} \times 2 \mathrm{C}$ & 122.7 & 202.1 & 83.0 & & $\mathrm{SO} \mathrm{X} 2 \mathrm{C}$ & -433.3 & -669.5 & -315.3 \\
\hline & SO DLU & 122.7 & 202.1 & 83.0 & & SO DLU & -433.3 & -669.5 & -315.8 \\
\hline & $4 \mathrm{c}$ & 123 & 203 & 83 & & $4 \mathrm{c}$ & -437 & -676 & -318 \\
\hline & Expt. & 145 & 227 & 103 & & Expt. & -488 & -743 & -360 \\
\hline \multirow[t]{7}{*}[\mathrm{MoOF}_{5}]{$^{2-}$} & $\mathrm{SR} X 2 \mathrm{C}$ & 142.2 & 217.7 & 104.4 & \multirow[t]{7}{*}[\mathrm{ReNF}_{4}]{$^{-}$} & $\mathrm{SR} \times 2 \mathrm{C}$ & -1482.2 & -1967.0 & -1239.7 \\
\hline & SR DLU & 142.2 & 217.7 & 104.5 & & SR DLU & -1482.2 & -1967.1 & -1239.7 \\
\hline & $\mathrm{SO} \times 2 \mathrm{C} P C$ & 164.6 & 256.8 & 118.5 & & $\mathrm{SO} \times 2 \mathrm{C} P C$ & -1793.8 & -2756.3 & -1312.5 \\
\hline & $\mathrm{SO} \times 2 \mathrm{C}$ & 164.7 & 257.0 & 118.6 & & $\mathrm{SO} \mathrm{X} 2 \mathrm{C}$ & -1795.7 & -2758.9 & -1314.2 \\
\hline & SO DLU & 164.7 & 257.0 & 118.6 & & SO DLU & -1795.7 & -2758.9 & -1314.2 \\
\hline & $4 \mathrm{c}$ & 166 & 259 & 119 & & $4 \mathrm{c}$ & -1788 & -2753 & -1306 \\
\hline & Expt. & 183 & 279 & 135 & & Expt. & -2117 & -3079 & -1637 \\
\hline \multirow{7}{*}[\mathrm{MoOBr}_{5}]{$^{2-}$} & $\mathrm{SR} \times 2 \mathrm{C}$ & 103.8 & 169.8 & 70.8 & \multirow[t]{7}{*}[\mathrm{ReNCl}_{4}]{$^{-}$} & $\mathrm{SR} \times 2 \mathrm{C}$ & -1003.4 & -1429.2 & -790.5 \\
\hline & SR DLU & 103.8 & 169.8 & 70.8 & & SR DLU & -1003.0 & -1428.8 & -790.1 \\
\hline & $\mathrm{SO} \times 2 \mathrm{C} P C$ & 112.8 & 176.2 & 81.1 & & $\mathrm{SO} \times 2 \mathrm{C} P C$ & -1229.4 & -1996.5 & -845.8 \\
\hline & $\mathrm{SO} \mathrm{X} 2 \mathrm{C}$ & 112.9 & 176.4 & 81.2 & & $\mathrm{SO} \mathrm{X} 2 \mathrm{C}$ & -1231.1 & -1998.7 & -847.2 \\
\hline & SO DLU & 112.9 & 176.4 & 81.2 & & SO DLU & -1230.7 & -1998.4 & $-846 . \mathrm{s}$ \\
\hline & $4 \mathrm{c}$ & 114 & 178 & 82 & & $4 \mathrm{c}$ & -1225 & -1994 & -841 \\
\hline & Expt. & 128 & 184 & 99 & & Expt. & -1544 & -2263 & -1184 \\
\hline \multirow[t]{7}{*}[\mathrm{WOCl}_{4}]{$^{-}$} & $\mathrm{SR} \times 2 \mathrm{C}$ & -142.4 & -212.2 & -107.5 & \multirow[t]{7}{*}[\operatorname{ReNBr}_{4}]{$^{-}$} & $\mathrm{SR} X 2 \mathrm{C}$ & -854.1 & -1268.3 & -647.1 \\
\hline & SR DLU & -142.4 & -212.2 & -107.4 & & SR DLU & -853.9 & -1268.1 & -646.8 \\
\hline & $\mathrm{SO} \times 2 \mathrm{C} P C$ & 193.2 & 316.1 & 131.8 & & $\mathrm{SO} \times 2 \mathrm{C} P C$ & -1010.7 & -1645.3 & -693.4 \\
\hline & $\mathrm{SO} \times 2 \mathrm{C}$ & -193.5 & -316.4 & -132.0 & & $\mathrm{SO} \times 2 \mathrm{C}$ & -1012.3 & -1647.3 & -694.8 \\
\hline & SO DLU & -193.5 & -316.4 & -132.0 & & SO DLU & -1012.0 & -1647.2 & -694.5 \\
\hline & $4 c$ & -193 & -316 & -131 & & $4 c$ & -1009 & -1646 & -691 \\
\hline & Expt. & - & - & - & & Expt. & -1340 & -1994 & -1013 \\
\hline$\left[\mathbf{W O F}_{5}\right]^{2-}$ & $\mathrm{SR} X 2 \mathrm{C}$ & -220.8 & -298.7 & -181.8 & {$\left[\mathrm{ReOBr}_{4}\right]$} & $\mathrm{SR} X 2 \mathrm{C}$ & -553.5 & -959.4 & -350.6 \\
\hline & SR DLU & -220.8 & -298.7 & -181.8 & & SR DLU & -553.6 & -959.4 & -350.6 \\
\hline & $\mathrm{SO} X 2 \mathrm{C} \mathrm{PCC}$ & 293.8 & 439.3 & 221.1 & & $\mathrm{SO} X 2 \mathrm{C}$ PCC & -735.9 & -1195.7 & -505. \\
\hline & $\mathrm{SO} \times 2 \mathrm{C}$ & -294.1 & -439.7 & -221.3 & & $\mathrm{SO} \times 2 \mathrm{C}$ & -737.3 & -1197.6 & -507.1 \\
\hline & SO DLU & -294.1 & -439.7 & -221.3 & & SO DLU & -737.3 & -1197.7 & -507.1 \\
\hline & $4 \mathrm{c}$ & -293 & -420 & -221 & & $4 c$ & -733 & -1197 & -501 \\
\hline & Expt. & -331 & -496 & -262 & & Expt. & - & - & - \\
\hline$\left[\mathbf{W O B r}_{5}\right]^{2-}$ & $\mathrm{SR} \times 2 \mathrm{C}$ & -116.6 & -183.0 & -83.4 & {$\left[\mathrm{ReOF}_{5}\right]^{-}$} & $\mathrm{SR} \times 2 \mathrm{C}$ & -1162.3 & -1651.0 & -917.8 \\
\hline & SR DLU & -116.6 & -183.0 & -83.3 & & SR DLU & -1162.4 & -1651.1 & -918.1 \\
\hline & $\mathrm{SO} \times 2 \mathrm{C} \mathrm{PCC}$ & -167.1 & -278.7 & -111.3 & & $\mathrm{SO} \times 2 \mathrm{C} P C \mathrm{C}$ & -1604.4 & -2469.0 & -1172.1 \\
\hline & $\mathrm{SO} \times 2 \mathrm{C}$ & -167.3 & -279.0 & -111.5 & & $\mathrm{SO} \times 2 \mathrm{C}$ & -1606.2 & -2471.4 & -1173.6 \\
\hline & SO DLU & -167.3 & -279.0 & -111.5 & & SO DLU & -1606.3 & -2471.6 & -1173.7 \\
\hline & $4 \mathrm{c}$ & -167 & -279 & -111 & & $4 c$ & -1600 & -2466 & -1166 \\
\hline & Expt. & - & - & - & & Expt. & -1959 & -2878 & -1499 \\
\hline$\left[\mathrm{OsOF}_{5}\right]$ & $\mathrm{SR} \times 2 \mathrm{C}$ & -370.9 & -541.6 & -285.6 & & & & & \\
\hline & SR DLU & -371.0 & -541.6 & -285.6 & & & & & \\
\hline & $\mathrm{SO} \times 2 \mathrm{C} P C$ & -518.5 & -811.2 & -372.2 & & & & & \\
\hline & $\mathrm{SO} \times 2 \mathrm{C}$ & -519.2 & -812.1 & -372.8 & & & & & \\
\hline & SO DLU & -519.3 & -812.1 & -372.8 & & & & & \\
\hline & $4 \mathrm{c}$ & -517 & -811 & -370 & & & & & \\
\hline & Expt. & -627 & -935 & -480 & & & & & \\
\hline
\end{tabular}


We note that neglecting these derivatives may result in error cancellation compared to the 4c results due to the picture-change effects of the two-electron integrals. From a formal point of view, a rigorous treatment of two-electron picture-change effects ${ }^{201}$ together with the decoupling derivatives should result in an excellent agreement with the DKS approach. However, this also increases the computational demands for the $2 \mathrm{c}$ ansatz, as it requires modifications of the two-electron infrastructure. Within the DLU scheme, the computational costs for the one-electron response and Sylvester equations to calculate the derivatives of $\boldsymbol{X}$ and $\boldsymbol{R}$ are drastically reduced. Therefore, they can be included with negligible computational overhead.

\subsection{Assessment of Basis Sets}

As the central idea of $\mathrm{X} 2 \mathrm{C}$ is to decouple the positive and the negative energy subspace after the introduction of a basis set, a proper assessment of various relativistic basis sets is in order. Here, we consider fully decontracted and segmented-contracted basis sets of various cardinal numbers and assess their impact on isotropic hyperfine coupling constants for 16 of the 17 transition-metal complexes. $\left[\mathrm{WOF}_{5}\right]^{2-}$ is neglected in the statistical evaluation due to convergence issues for the alignment of spin $x$ and $y$ with the ET basis. The MAPD and STD are shown in Fig. 1.

First, all decontracted basis sets possess small errors - typically in the range of 1-3\% in MAPD. The ANO-R basis shows the smallest mean errors with $0.37 \%$ and a standard deviation of $0.24 \%$. Very similar errors are found for the quadruple- $\zeta$ combinations of the correlation-consistent (cc, ccJ) and the Dyall basis sets. Here, the MAPDs amount to 0.49\% for both combinations. However, this comes at the price of an increased dimension of the involved matrices. Even the comparably small IGLO-II/Dyall-VDZ combination features more basis functions than the segmented-contracted triple- $\zeta$ bases, see Tab. 1. As the different basis sets for the light atoms do not substantially affect the HFC constant of the

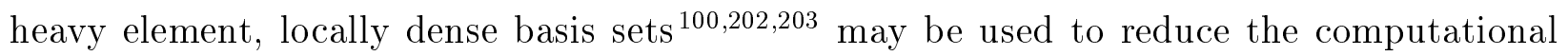


Figure 1: Assessment of various basis sets compared to an even-tempered reference for 16 of the 17 transition-metal complexes. $\left[\mathrm{WOF}_{5}\right]^{2-}$ is neglected in the statistical evaluation due to convergence issues for the alignment of spin $x$ and $y$ with the ET basis. MAPD and STD denote the mean absolute percent-wise error and its standard deviation. For brevity, the suffix "unc" is omitted for the combinations of the Dyall basis and the ANO basis sets. See Tab. 1 for further details on the basis sets and their size.

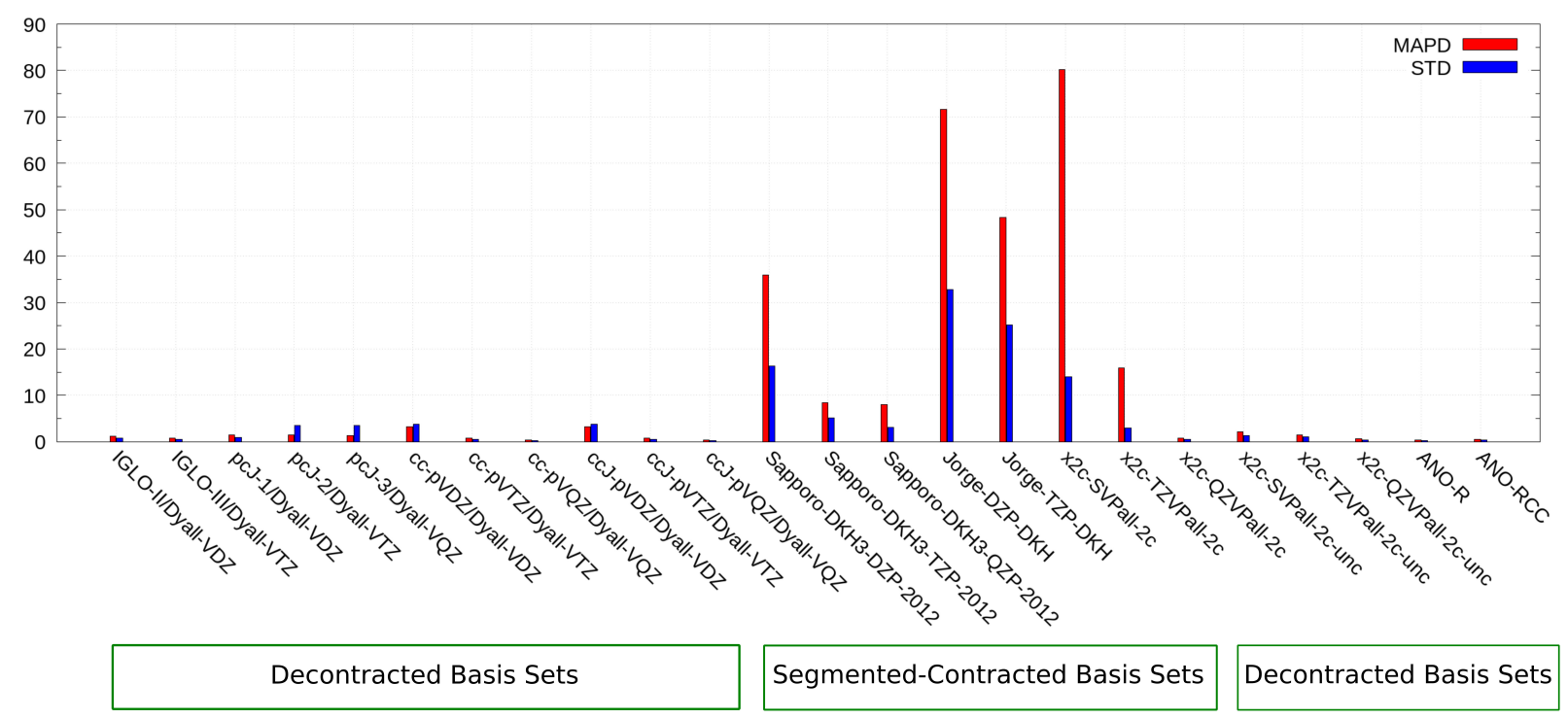

demands, i.e. the large basis set is only used for the heavy element and a basis set of a decreased cardinal number is employed for the light element.

Second, the segmented-contracted double- $\zeta$ basis sets feature notably large errors. Here, the Sapporo-DKH3-DZP-2012 bases result in an MAPD of 35.93\% and the Jorge-DKH-DZP or x2c-SVPall-2c bases result in MAPDs of $70.91 \%$ and $80.83 \%$, respectively. These basis sets are thus clearly insufficient for accurate calculations of hyperfine coupling constants. Triple- $\zeta$ basis sets are a major improvement in this regard and the errors are reduced to 8-16\% with the Sapporo and x2c-type bases. Notably, the Jorge-DKH-TZP basis still leads to a large MAPD of $48.56 \%$. The quadruple- $\zeta$ Sapporo and x2c-type basis sets perform on par with the fully decontracted basis sets when comparing both the errors and number of functions. The x2c-QZVPall-2c and Sapporo-DKH3-QZP-2012 basis sets employ 8799 and 9804 functions for the 17 compounds. For the uncontracted Dyall basis sets, about 6500 (double- $\zeta$ ), 9600 (triple- $\zeta$ ), and 12000 (quadruple- $\zeta$ ) functions are used throughout the molecular set. 
Decontracting the x2c-QZVPall-2c basis does not significantly alter the results, and consequently this segmented-contracted basis set is sufficiently flexible - in contrast to the double and triple- $\zeta$ basis sets. Decontracting the latter bases results in a major improvement and the MAPDs are in the same range as those of the uncontracted Dyall and ANO basis sets.

Overall, these findings confirm our previous studies for a smaller set of molecules and the scalar-relativistic approach. ${ }^{123}$ Compared to other magnetic properties such as NMR shielding constants, $\frac{115[144}{1}$ the good performance of the Sapporo basis sets is remarkable. This is further notable as these basis sets are optimized with the point charge model and finite nucleus size effects may be pronounced for the hyperfine coupling interaction of heavy elements. $.32|204| 205$

\subsection{Assessment of Density Functional Approximations}

Furthermore, we assessed the accuracy of a variety of pure, hybrid, and range-separated hybrid (RSH) density functional approximations in predicting the isotropic hyperfine coupling constants with the spin-orbit DLU-X2C Hamiltonian for a subset of 12 transition-metal complexes from the 4c study: $\left[\mathrm{MoOCl}_{4}\right]^{-},\left[\mathrm{MoOF}_{5}\right]^{2-},\left[\mathrm{MoOBr}_{4}\right]^{2-},\left[\mathrm{WOF}_{5}\right]^{2-},\left[\mathrm{TcNF}_{4}\right]^{-}$, $\left[\mathrm{TcNCl}_{4}\right]^{-},\left[\mathrm{TcNBr}_{4}\right]^{-},\left[\mathrm{ReNF}_{4}\right]^{-},\left[\mathrm{ReNCl}_{4}\right]^{-},\left[\mathrm{ReNBr}_{4}\right]^{-},\left[\mathrm{ReOF}_{5}\right]^{-}$, and $\left[\mathrm{OsOF}_{5}\right]$. The resulting MAPDs and STDs when compared to experimental reference data are visualized in Fig. 2. Note that Harteee-Fock theory results in an MAPD of more than $100 \%$ and is clearly insufficient for these transition-metal complexes.

Unsurprisingly, pure density functionals without Hartree-Fock exchange performed the poorest in the study, with MAPDs exceeding 20\% for KT3, TPSS, PBE, BP86, and S-VWN. The $\mathrm{r}^{2} \mathrm{SCAN}$ functional represents a notable exception to this with an MAPD of $16.76 \%$ and STD of 13.08\%, outperforming some hybrid and RSH functionals such as B3LYP, B97, revTPSSh, and CAM-QTP-00. Despite being a hybrid, B3LYP performed rather poorly with an MAPD of $19.50 \%$. The range-separated version CAM-B3LYP improves on this result marginally, but still falls in the latter half of functionals in ranking and is also the 
Figure 2: Assessment of various density functional approximations compared to the experimental findings for a subset of 12 transition-metal complexes. MAPD and STD denote the mean absolute percent-wise error and its standard deviation. Note that HF shows an MAPD and a STD of $122.10 \%$ and $55.23 \%$, respectively. Therefore, the HF results are omitted.

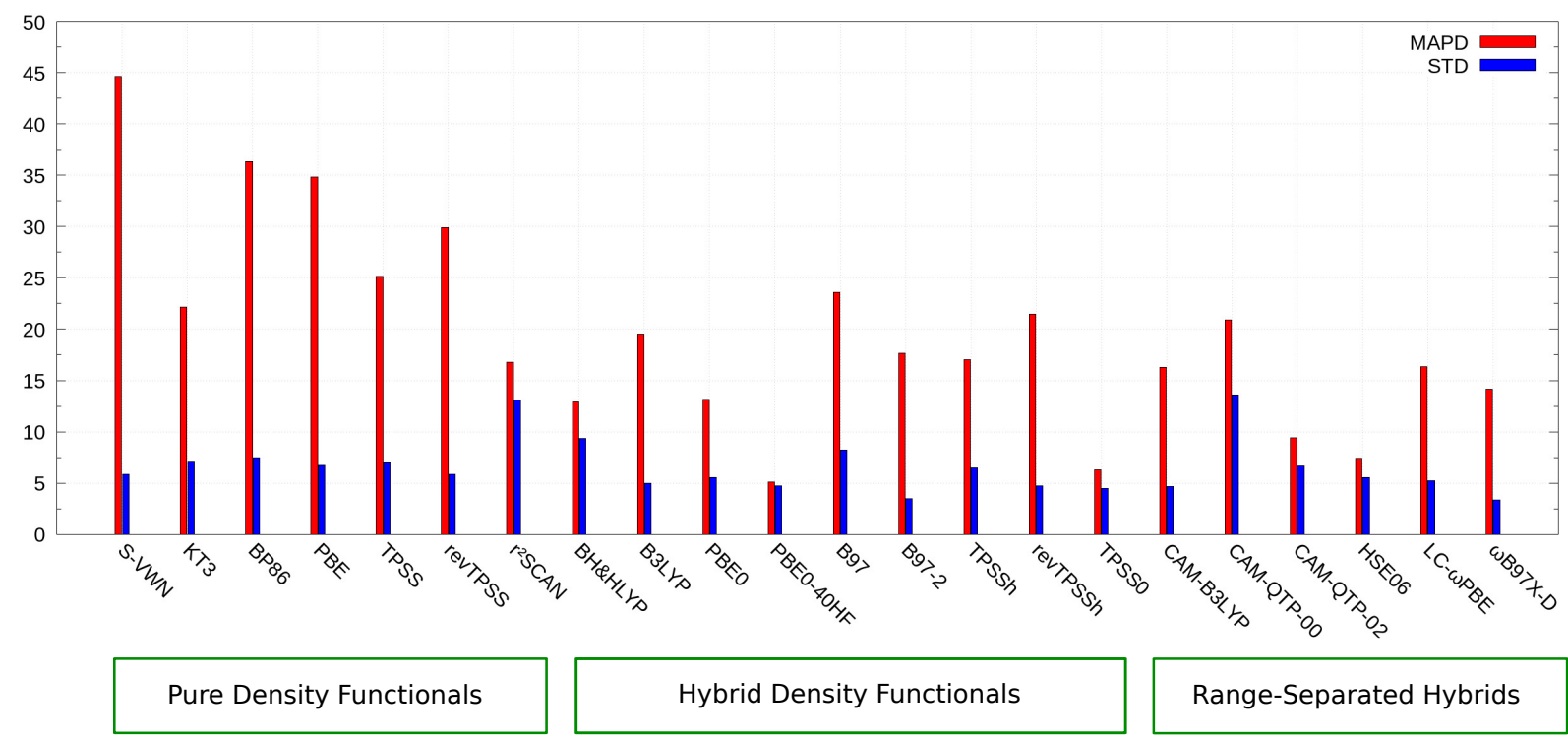

second worst RSH tested, with the first being CAM-QTP-00.

A clear trend that can be observed from the results is that functionals with HF exchange seemed to yield more accurate isotropic hyperfine coupling constants, which is evident in 9 of the top 10 functionals being (range-separated) hybrids. In particular, the modification of the PBE0 functional with $40 \% \mathrm{HF}$ exchange produced the best MAPD and STD values by a considerable margin for this subset of transition metal complexes, with the next most accurate functionals being TPSS0, HSE06, and CAM-QTP-02. However, this large amount of HF exchange in PBE0-40HF may negatively affect other properties and PBE0 may be a more robust functional for general chemical properties. Interestingly, the use of a range separated over global hybrid scheme did not systematically improve the results, and the BH\&HLYP and PBE0 functionals perform comparably well. These results agree with a recent functional study of magnetizabilities for 28 small molecules ${ }^{206 \mid 207}$ and also other benchmark studies on magnetic properties $\frac{208}{214}$ suggesting that inclusion of HF exchange is important for magnetic properties in general. These conclusions also hold for both spin-orbit DLU-X2C and 
previously reported ZORA results ${ }^{\sqrt{12}}$ for the hyperfine coupling constants of small mercury compounds as shown in Table S2 of the Supporting Information.

\section{Assessment of Efficiency and Further Studies}

To test our implementation on a larger molecule, we study $\left[\mathrm{Pt}\left(\mathrm{C}_{6} \mathrm{Cl}_{5}\right)_{4}\right]^{-}$consisting of 45 atoms depicted in Fig. 3. The isotropic HFC constant and the principal components are listed in Tab. 3 at various levels of theory. Quasi-relativistic calculations are only performed for the hybrid functionals as the pure density functionals yield scalar-relativistic results with a comparably large deviation towards experimental findings. Note that we use the RI- $J$ approximation in the $2 \mathrm{c}$ calculations to increase the efficiency of the approach. For the SCF procedure, the auxiliary basis set of the RI- $J$ approximation needs to fit the electron

density. ${ }^{1092151216}$ Consequently, the universal (uncontracted) x2c-type fitting bases ${ }^{1061144}$ are a reasonable choice for the uncontracted Dyall-VTZ/pcJ-2 orbital bases. This differs significantly from the application of RI- $J$ to post-Hartree-Fock or post-Kohn-Sham ansätze and response properties. ${ }^{217} \sqrt{220}$ Here, the product of orbitals needs to be modeled by the auxiliary bases. Indeed, the scalar-relativistic calculations of the Dyall-VTZ/pcJ-2 basis confirmed that the RI- $J$ approximation can be used with the universal x2c-type fitting basis sets. The errors from the RI- $J$ approximation are smaller than those from neglecting the derivatives of $\boldsymbol{X}$ and $\boldsymbol{R}$ as well as the DLU error according to Sec. 5.1. The x2c-QZVPall-2c bases are combined with tailored auxiliary bases. ${ }^{144}$

Overall, the Dyall-VTZ/pcJ-2 and x2c-QZVPall-2c basis sets lead to similar results and a large amount of HF exchange is generally advantageous for accurate HFC constants. Spinorbit effects are of great importance for the principal components of the HFC tensor, whereas the impact on the isotropic constant is comparably small and typically amounts to 50$100 \mathrm{MHz}$. The pure density functionals significantly underestimate the HFC constant with $\mathrm{r}^{2} \mathrm{SCAN}$ again representing a notable exception. Yet, it is still outperformed by all hybrid 
Figure 3: Molecular structure of $\left[\mathrm{Pt}\left(\mathrm{C}_{6} \mathrm{Cl}_{5}\right)_{4}\right]^{-}$. Colors: $\mathrm{Pt}$ yellow, $\mathrm{Cl}$ green, $\mathrm{C}$ grey.

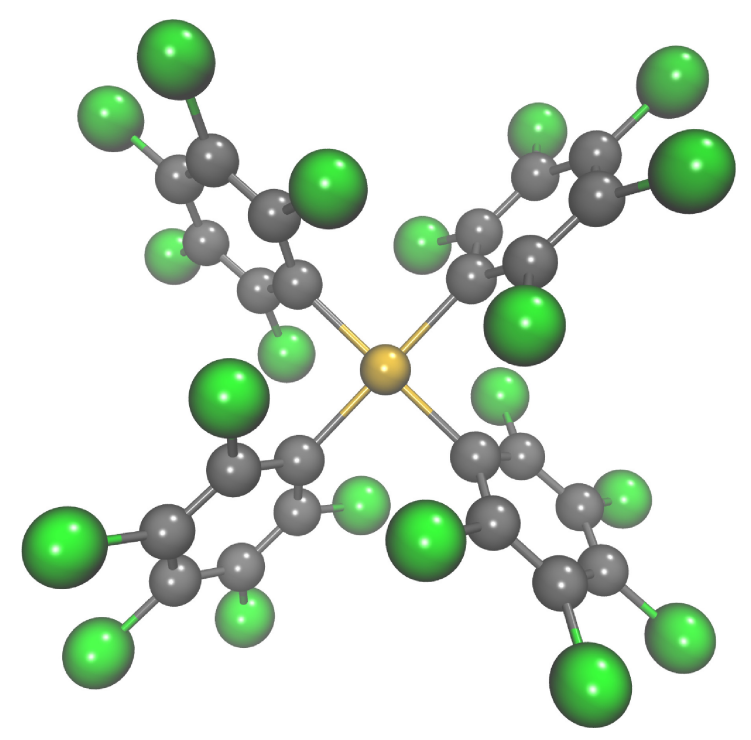

functionals. B3LYP, PBE0, and TPSSh overestimate the HFC constant by 500-700 MHz. BH\&HLYP and PBE0-40HF result in a good agreement with the experimental findings. These two functionals perform best among the global hybrids similar to Sec. 5.3. Also, the range-separated functional LC- $\omega \mathrm{PBE}$ leads to better results than the related PBE0 functional. In contrast, CAM-B3LYP does not substantially improve upon B3LYP. This confirms that range separation is not an a priori improvement, despite a comparably large amount of HF exchange being necessary. This suggests that more sophisticated functionals such as local hybrid functionals, ${ }^{222}$ featuring a position-dependent admixture of HF exchange, may be useful for the HFC constant. A simple local mixing function based on the iso-orbital indicator $^{\sqrt{222}}$ is generally not sufficient for the HFC of transition metals ${ }^{\sqrt{223}}$ and more elaborate approaches ${ }^{2081224225}$ may be helpful. A first ansatz with Johnson's local hybrid functional based on the correlation length ${ }^{224}$ yields a scalar-relativistic HFC of $7165 \mathrm{MHz}$ (Dyall-VTZ/pcJ-2 basis set, grid 3a). Note that we used the seminumerical exchange methodology for the evaluation of the respective XC terms ${ }^{208}$ as outlined by Plessow and Weigend. ${ }^{104}$ Similar to our recent benchmark studies of the NMR coupling constants, $\stackrel{208}{\omega B}$ B7X-D performs best for the full spin-orbit DLU-X2C, RI- $J$ method in predicting the principal components of the hyperfine coupling constant. 
Table 3: Principal components of the hyperfine coupling constant and isotropic value in MHz. Scalar-relativistic (SR) calculations are carried out with the exact Coulomb integrals and the RI- $J$ method. Two-component spin-orbit (SO) calculations only use the latter and the mSNSO approximation. Experimental (Expt.) results are taken from Ref. 221.

\begin{tabular}{|c|c|c|c|c|c|c|c|c|c|}
\hline \multirow[b]{2}{*}{ Functional } & \multirow[b]{2}{*}{ Hamiltonian } & \multicolumn{4}{|c|}{ Dyall-VTZ-unc/pcJ-2-unc } & \multicolumn{4}{|c|}{ x2c-QZVPall-2c } \\
\hline & & $A_{\text {iso }}$ & $A_{11}$ & $A_{22}$ & $A_{33}$ & $A_{\text {iso }}$ & $A_{11}$ & $A_{22}$ & $A_{33}$ \\
\hline \multirow[t]{2}{*}{ KT3 } & SR DLU & 6106 & 5735 & 6255 & 6328 & 5951 & 5579 & 6089 & 6184 \\
\hline & SR DLU, RI- $J$ & 6107 & 5736 & 6255 & 6330 & 5945 & 5574 & 6083 & 6179 \\
\hline \multirow[t]{2}{*}{ BP86 } & SR DLU & 5317 & 4943 & 5432 & 5576 & 5193 & 4822 & 5298 & 5458 \\
\hline & SR DLU, RI- $J$ & 5316 & 4942 & 5431 & 5576 & 5192 & 4821 & 5298 & 5458 \\
\hline \multirow[t]{2}{*}{ PBE } & SR DLU & 5582 & 5204 & 5715 & 5826 & 5441 & 5066 & 5564 & 5694 \\
\hline & SR DLU, RI- $J$ & 5579 & 5202 & 5712 & 5824 & 5439 & 5064 & 5561 & 5692 \\
\hline \multirow[t]{2}{*}{ TPSS } & SR DLU & 6006 & 5626 & 6154 & 6237 & 5879 & 5500 & 6016 & 6120 \\
\hline & SR DLU, RI- $J$ & 6002 & 5622 & 6150 & 6234 & 5876 & 5497 & 6014 & 6118 \\
\hline \multirow{2}{*}{$\mathrm{r}^{2} \mathrm{SCAN}$} & SR DLU & 8459 & 8238 & 8498 & 8641 & 8493 & 8277 & 8530 & 8673 \\
\hline & SR DLU, RI- $J$ & 8465 & 8245 & 8504 & 8648 & 8487 & 8269 & 8527 & 8666 \\
\hline \multirow[t]{3}{*}{ BH\&HLYP } & SR DLU & 7083 & 6890 & 7048 & 7311 & 7093 & 6900 & 7060 & 7320 \\
\hline & SR DLU, RI- $J$ & 7089 & 6896 & 7054 & 7317 & 7100 & 6907 & 7066 & 7326 \\
\hline & SO DLU, RI- $J$ & 7240 & 6327 & 7689 & 7705 & 7246 & 6335 & 7695 & 7707 \\
\hline \multirow[t]{3}{*}{ B3LYP } & SR DLU & 8087 & 7787 & 8105 & 8367 & 8091 & 7790 & 8112 & 8369 \\
\hline & SR DLU, RI- $J$ & 8078 & 7775 & 8101 & 8357 & 8089 & 7787 & 8113 & 8367 \\
\hline & SO DLU, RI- $J$ & 7950 & 7131 & 8262 & 8456 & 7949 & 7134 & 8264 & 8450 \\
\hline \multirow[t]{3}{*}{ PBE0 } & SR DLU & 7893 & 7673 & 7851 & 8155 & 7901 & 7681 & 7860 & 8161 \\
\hline & SR DLU, RI- $J$ & 7888 & 7668 & 7846 & 8150 & 7906 & 7686 & 7866 & 8167 \\
\hline & SO DLU, RI- $J$ & 7871 & 7029 & 8257 & 8327 & 7871 & 7033 & 8259 & 8320 \\
\hline \multirow[t]{3}{*}{ PBE0-40HF } & SR DLU & 7313 & 7129 & 7268 & 7542 & 7327 & 7143 & 7283 & 7554 \\
\hline & SR DLU, RI- $J$ & 7319 & 7136 & 7274 & 7548 & 7333 & 7150 & 7289 & 7560 \\
\hline & SO DLU, RI- $J$ & 7482 & 6590 & 7920 & 7935 & 7485 & 6595 & 7924 & 7935 \\
\hline \multirow[t]{3}{*}{ TPSSh } & SR DLU & 8077 & 7819 & 8073 & 8340 & 8101 & 7844 & 8097 & 8360 \\
\hline & SR DLU, RI- $J$ & 8077 & 7817 & 8075 & 8339 & 8089 & 7826 & 8091 & 8346 \\
\hline & SO DLU, RI- $J$ & 8000 & 7283 & 8345 & 8373 & 8002 & 7291 & 8263 & 8452 \\
\hline \multirow[t]{3}{*}{ TPSS0 } & SR DLU & 7696 & 7493 & 7652 & 7942 & 7717 & 7515 & 7675 & 7961 \\
\hline & SR DLU, RI- $J$ & 7702 & 7500 & 7657 & 7948 & 7723 & 7522 & 7681 & 7967 \\
\hline & SO DLU, RI- $J$ & 7815 & 7018 & 8208 & 8218 & 7824 & 7029 & 8212 & 8231 \\
\hline \multirow[t]{3}{*}{ CAM-B3LYP } & SR DLU & 7803 & 7546 & 7754 & 8109 & 7811 & 7555 & 7763 & 8115 \\
\hline & SR DLU, RI- $J$ & 7808 & 7552 & 7759 & 8114 & 7816 & 7560 & 7769 & 8121 \\
\hline & SO DLU, RI- $J$ & 7696 & 6800 & 8104 & 8184 & 7699 & 6802 & 8106 & 8189 \\
\hline \multirow[t]{3}{*}{ CAM-QTP-00 } & SR DLU & 6648 & 6465 & 6611 & 6868 & 6657 & 6475 & 6621 & 6876 \\
\hline & SR DLU, RI- $J$ & 6654 & 6471 & 6618 & 6874 & 6664 & 6482 & 6628 & 6883 \\
\hline & SO DLU, RI- $J$ & 6909 & 5951 & 7375 & 7400 & 6916 & 5960 & 7382 & 7406 \\
\hline \multirow[t]{3}{*}{ CAM-QTP-02 } & SR DLU & 7141 & 6905 & 7090 & 7429 & 7148 & 6912 & 7098 & 7435 \\
\hline & SR DLU, RI- $J$ & 7147 & 6912 & 7096 & 7435 & 7155 & 6919 & 7104 & 7441 \\
\hline & SO DLU, RI- $J$ & 7178 & 6219 & 7629 & 7685 & 7177 & 6222 & 7632 & 7677 \\
\hline \multirow[t]{3}{*}{ HSE06 } & SR DLU & 7749 & 7520 & 7715 & 8011 & 7757 & 7529 & 7726 & 8018 \\
\hline & SR DLU, RI- $J$ & 7752 & 7522 & 7720 & 8014 & 7762 & 7533 & 7731 & 8023 \\
\hline & SO DLU, RI- $J$ & 7720 & 6905 & 8089 & 8166 & 7722 & 6908 & 8089 & 8168 \\
\hline \multirow[t]{3}{*}{$\mathrm{LC}-\omega \mathrm{PBE}$} & SR DLU & 7315 & 7099 & 7231 & 7615 & 7319 & 7103 & 7236 & 7618 \\
\hline & SR DLU, RI- $J$ & 7321 & 7105 & 7237 & 7621 & 7325 & 7109 & 7243 & 7624 \\
\hline & SO DLU, RI- $J$ & 7438 & 6548 & 7872 & 7894 & 7435 & 6546 & 7869 & 7889 \\
\hline$\omega \mathrm{B} 97 \mathrm{X}-\mathrm{D}$ & SR DLU & 7196 & 6930 & 7169 & 7488 & 7194 & 6927 & 7168 & 7485 \\
\hline & SR DLU, RI- $J$ & 7201 & 6935 & 7175 & 7494 & 7200 & 6933 & 7174 & 7491 \\
\hline & SO DLU, RI- $J$ & 7270 & 6421 & 7639 & 7749 & 7265 & 6419 & 7634 & 7742 \\
\hline Expt. & & 7322 & 6375 & 7735 & 7855 & 7322 & 6375 & 7735 & 7855 \\
\hline
\end{tabular}

We note that the 2c DLU-X2C/PBE0 calculation of one spin contribution to the HFC matrix of all atoms finished in 5.5 to 6 hours for the Dyall-VTZ/pcJ-2 basis set (79-85 SCF iterations based on converged UKS orbitals) on 24 OpenMP threads of an Intel ${ }^{\circledR}$ Xeon ${ }^{\circledR}$ Gold 
6212U CPU @ 2.40 GHz (code was compiled with Intel ${ }^{\circledR}$ Fortran Compiler 19.0.1.144). For the x2c-QZVPall-2c bases, the calculations take 18.5 to 24.5 hours (70-103 SCF iterations). For comparison, the wall time with 12 OpenMP threads amounts to 26.5 to 41.9 hours for 64-106 SCF iterations. The calculation of the respective derivatives of the one-electron Hamiltonian for the HFC matrix of all atoms amounts to 15.4 minutes and 41.7 minutes for Dyall-VTZ/pcJ-2 and x2c-QZVPall-2c with the DLU scheme (24 threads). In comparison, the full X2C ansatz takes 630 minutes and 1851 minutes, respectively. Hence, the speed-up by the DLU scheme amounts to a factor of 41 and 44 . Further speed-ups are possible by selecting the corresponding nuclei of interest similar to NMR coupling constants $\sqrt{55 \mid 100}$ and NMR chemical shifts. ${ }^{213}$ This way the wall time for the DLU-X2C Hamiltonian derivatives is reduced to 0.3 (Dyall-VTZ/pcJ-2) and 0.9 minutes (x2c-QZVPall-2c); a speed-up by a factor of about 45-50 is observed. Thus, the efficiency of our implementation is clearly demonstrated and the derivatives of the decoupling can routinely be included in DLU-X2C calculations. The computation time is clearly determined by the two-electron integrals in

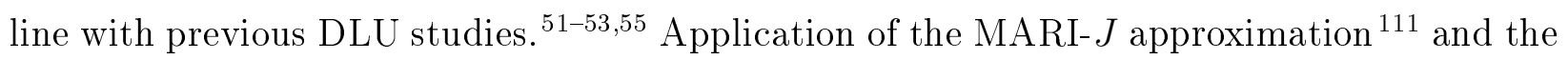
seminumerical exchange approximation $\frac{104 \mid 105}{10}$ reduces the time for the two-electron integrals. However, it increases the number of SCF iterations for the Dyall-VTZ/pcJ-2 basis set from 79-85 to 82-130. The wall time amounts to 4.5-6.9 hours while changing the isotropic HFC constant by $-5 \mathrm{MHz}$.

\section{Application to Rare-Earth Single Molecule Magnets}

Recently, the discovery of a series of $\mathrm{La}(\mathrm{II})$ and $\mathrm{Lu}(\mathrm{II})$-based single molecule magnets: $\left[\mathrm{La}\left(\mathrm{OAr}^{*}\right)_{3}\right]^{1-}(\mathbf{1}),\left[\mathrm{Lu}\left(\mathrm{NR}_{2}\right)_{3}\right]^{1-}(\mathbf{2})$, and $\left[\mathrm{Lu}\left(\mathrm{OAr}^{*}\right)_{3}\right]^{1-}(\mathbf{3})\left(\mathrm{OAr}^{*}=2,6-\mathrm{Ad}_{2}-4-\mathrm{t}-\mathrm{Bu}-\right.$ $\mathrm{C}_{6} \mathrm{H}_{2} \mathrm{O}, \mathrm{Ad}=$ adamantyl, $\mathrm{t}-\mathrm{Bu}=$ tert-butyl, $\mathrm{R}=\mathrm{SiMe}_{3}$ with $\mathrm{Me}=$ methyl) was reported..$^{22}$ The three molecules are depicted in Fig. 4. It was demonstrated through EPR and KS-DFT studies that variation of the $s$-orbital mixing present in the $6 s / d$ singly occupied molec- 
Figure 4: Molecular structures of $\left[\mathrm{La}\left(\mathrm{OAr}^{*}\right)_{3}\right]^{1-}$ (1) (left), $\left[\mathrm{Lu}\left(\mathrm{NR}_{2}\right)_{3}\right]^{1-}$ (2) (middle), and $\left[\mathrm{Lu}\left(\mathrm{OAr}^{*}\right)_{3}\right]^{1-}(\mathbf{3})$ (right), $\mathrm{OAr}^{*}=2,6-\mathrm{Ad}_{2}-4-\mathrm{t}-\mathrm{Bu}-\mathrm{C}_{6} \mathrm{H}_{2} \mathrm{O}, \mathrm{Ad}=$ adamantyl, t-Bu= tertbutyl, $\mathrm{R}=\mathrm{SiMe}_{3}$ with $\mathrm{Me}=$ methyl. $\mathrm{H}$ atoms are omitted for clarity. Colors: La cyan, $\mathrm{O}$ red, C grey, Lu pink, N blue, Si brown.
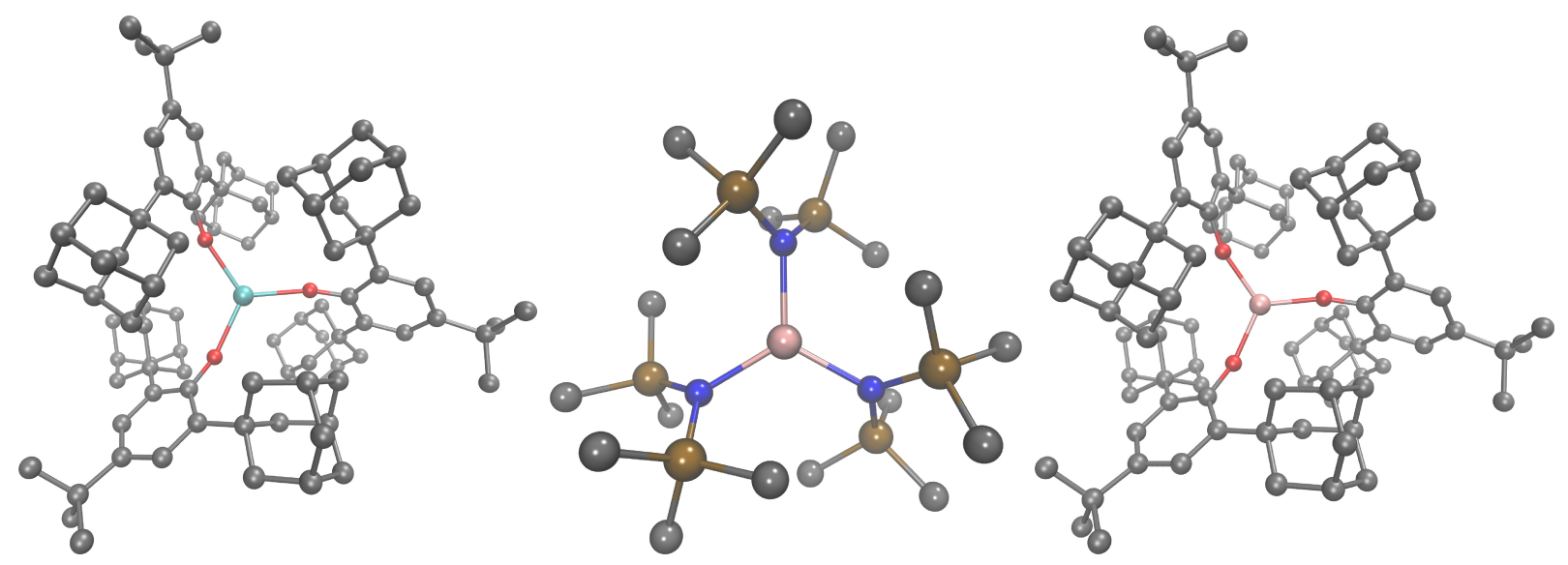

ular orbital (SOMO) of these complexes could be used to tune their magnetic structure, with $\left[\mathrm{Lu}(\mathrm{OAr})_{3}\right]^{-}$representing a desirable orbital configuration through its possession of a significant hyperfine clock transition of approximately $9 \mathrm{GHz}$ and its enhanced magnetic relaxation times.

The scalar-relativistic DFT method used to approximate the hyperfine coupling matrix in this study qualitatively described the expected increase in isotropic hyperfine coupling constant with larger $6 s$ character of the SOMO (arising from the Fermi-contact interaction). However, the values themselves possessed errors of roughly one order of magnitude when compared with experimental results, mostly attributed to the non-relativistic operator used to calculate the HFC matrix giving rise to a large picture-change error ${ }^{15}$ Herein, this method will be denoted SR-NR-DFT. While such a method proved useful when paired with experiment, improvements which can achieve quantitative accuracy are desired if in silico discovery of optimal SMMs is to be realized.

To assess the potential improvement obtained from the present method, we applied our DFT-based X2C approach to compute the hyperfine coupling matrix for (1), (2), and (3), comparing with the SR-NR-DFT and experimentally determined EPR results. For direct comparison, the same computational methodology ${ }^{22}$ was used between the SR-NR-DFT and 
X2C calculations: The TPSS density functional ${ }^{158}$ was used with all-electron NMR-tailored x2c-TZVPPall-s basis sets for the lanthanide atom (Ln) and x2c-SVPall-s basis sets for the ligand atoms. .115 In addition to the parent $\mathrm{x} 2 \mathrm{c}$-type basis sets, these feature additional tight $p$ functions and minimal extension for the outer-core and inner valence region. The finite nucleus model and DLU were used for both scalar-relativistic (SR) and spin-orbit (SO) $\mathrm{X} 2 \mathrm{C}$ calculations. These calculations were also repeated for the spin-orbit $2 \mathrm{c}$ extensions of the x2c-type basis sets ${ }^{106}$ (x2c-TZVPPall-2c/Ln and x2c-SVPall-2c/H,C,N,O,Si). We also repeated the calculations using the $\mathrm{r}^{2} \mathrm{SCAN}, \frac{161162}{13 B}$ PB, ${ }^{131132}$ PBE0-40HF,

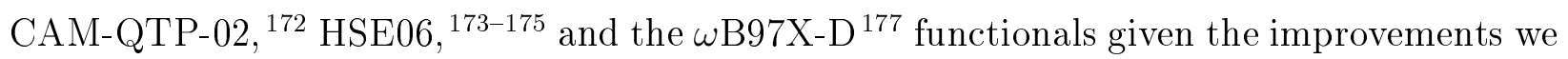
observed above with the $\mathrm{r}^{2} \mathrm{SCAN}$ and hybrid functionals. Lastly, we performed calculations using the $\mathrm{x} 2 \mathrm{c}-\mathrm{QZVPall}-2 \mathrm{c}$ basis set for the Ln atom $\frac{144}{10}$ to assess the accuracy of the method. The results are given in Tab. 4 .

Modest improvements are observed when going from the $\mathrm{x} 2 \mathrm{c}-\mathrm{s}$ to $\mathrm{x} 2 \mathrm{c}-2 \mathrm{c}$ type basis sets on the Ln atom for the SO methods, and also when using a hybrid or RSH as opposed to a pure functional. Here, the triple- $\zeta$ basis sets are already sufficient for the SO calculations as the application of the x2c-QZVPall-2c basis alters the HFC constants by about 20-30 MHz. Larger changes of up to $150 \mathrm{MHz}$ are found in the SR calculations. r $^{2}$ SCAN again outperforms TPSS which is consistent with the findings of Sections 5.3 and 6 . In some cases, the SR results for different basis set and functional configurations are closer to experiment than the SO results (e.g. x2c-TZVPPall-2c/PBE0-40HF), which is likely due to error cancellation. This can be posited from the inconsistent corrections in the SR results when going from pure to hybrid and RSH functionals, in contrast with a smoother convergence of the SO method. The PBE0-40HF and RSH functionals tested with x2c-TZVPPall-2c basis set perform similarly well when compared to experiment. The x2c-QZVPall-2c/wB97X-D configuration yields accurate results for both the SO and SR methods in all three compounds. For $\mathrm{SO}$ in particular, deviations from experiment are lowered to tens of $\mathrm{MHz}$ or less. Overall, the results demonstrate that the use of a relativistic hyperfine "contact" operator with finite 
nucleus corrects the majority of the picture-change error observed in SR-NR-DFT for these complexes.

Table 4: Hyperfine coupling constants calculated for Ln(II)-based single molecule magnets at various levels of theory and compared with experimental results (Expt.). ${ }^{22}$ For brevity, only the basis set of the Ln atom is given here. The x2c-SVPall-s and x2c-SVPall-2c basis set is employed for the light elements. The SR-NR results are taken from Ref. 22, All values are given in $\mathrm{MHz}$.

\begin{tabular}{|c|c|c|c|c|}
\hline Method & Hamiltonian & (1) & $(2)$ & (3) \\
\hline \multirow{3}{*}{ x2c-TZVPPall-s/TPSS } & SR-NR & 8274 & 17040 & 23950 \\
\hline & $\mathrm{SR}$ & 1746 & 2259 & 3192 \\
\hline & $\mathrm{SO}$ & 1716 & 2227 & 3139 \\
\hline \multirow[t]{2}{*}{ x2c-TZVPPall-2c/TPSS } & SR & 1747 & 2258 & 3177 \\
\hline & $\mathrm{SO}$ & 1726 & 2236 & 3151 \\
\hline \multirow{2}{*}{ x2c-QZVPall-2c/TPSS } & SR & 1746 & 2239 & 3168 \\
\hline & $\mathrm{SO}$ & 1724 & 2212 & 3139 \\
\hline \multirow[t]{2}{*}{$\mathrm{x} 2 \mathrm{c}-\mathrm{TZVPPall}-2 \mathrm{c} / \mathrm{r}^{2} \mathrm{SCAN}$} & SR & 1777 & 2372 & 3248 \\
\hline & $\mathrm{SO}$ & 1749 & 2322 & 3202 \\
\hline \multirow[t]{2}{*}{$\mathrm{x} 2 \mathrm{c}-\mathrm{QZVPall}-2 \mathrm{c} / \mathrm{r}^{2} \mathrm{SCAN}$} & SR & 1779 & 2375 & 3259 \\
\hline & $\mathrm{SO}$ & 1750 & 2323 & 3211 \\
\hline \multirow[t]{2}{*}{ x2c-TZVPPall-2c/PBE0 } & $\mathrm{SR}$ & 1756 & 2270 & 3194 \\
\hline & $\mathrm{SO}$ & 1804 & 2241 & 3241 \\
\hline \multirow[t]{2}{*}{ x2c-QZVPall-2c/PBE0 } & $\mathrm{SR}$ & 1839 & 2266 & 3266 \\
\hline & $\mathrm{SO}$ & 1823 & 2235 & 3252 \\
\hline \multirow[t]{2}{*}{ x2c-TZVPPall-2c/PBE0-40HF } & SR & 1843 & 2271 & 3300 \\
\hline & $\mathrm{SO}$ & 1809 & 2243 & 3261 \\
\hline \multirow{2}{*}{$\mathrm{x} 2 \mathrm{c}-\mathrm{QZVPall}-2 \mathrm{c} / \mathrm{PBE} 0-40 \mathrm{HF}$} & SR & 1889 & 2355 & 3445 \\
\hline & $\mathrm{SO}$ & 1837 & 2233 & 3277 \\
\hline \multirow[t]{2}{*}{ x2c-TZVPPall-2c/CAM-QTP-02 } & SR & 2080 & 2504 & 3499 \\
\hline & $\mathrm{SO}$ & 2040 & 2464 & 3459 \\
\hline \multirow[t]{2}{*}{ x2c-QZVPall-2c/CAM-QTP-02 } & $\mathrm{SR}$ & 2100 & 2495 & 3499 \\
\hline & $\mathrm{SO}$ & 2074 & 2463 & 3471 \\
\hline \multirow[t]{2}{*}{ x2c-TZVPPall-2c/HSE06 } & $\mathrm{SR}$ & 1824 & 2297 & 3291 \\
\hline & $\mathrm{SO}$ & 1795 & 2262 & 3249 \\
\hline \multirow[t]{2}{*}{ x2c-QZVPall-2c/HSE06 } & $\mathrm{SR}$ & 1838 & 2283 & 3290 \\
\hline & $\mathrm{SO}$ & 1817 & 2257 & 3260 \\
\hline \multirow[t]{2}{*}{ x2c-TZVPPall-2c/ $/$ B97X-D } & SR & 1901 & 2411 & 3501 \\
\hline & $\mathrm{SO}$ & 1870 & 2378 & 3459 \\
\hline \multirow[t]{2}{*}{ x2c-QZVPall-2c/ $\omega$ B97X-D } & SR & 1929 & 2489 & 3655 \\
\hline & $\mathrm{SO}$ & 1867 & 2359 & 3472 \\
\hline \multicolumn{2}{|l|}{ Expt. } & $1840 \pm 25$ & $2443 \pm 50$ & $3467 \pm 50$ \\
\hline
\end{tabular}


Calculation of the spin-orbit DLU-X2C Hamiltonian derivatives for the x2c/TZVPPall2c/TPSS calculations took roughly two minutes for (1) and (3) (ca. 2000 basis functions) and 16 seconds for (2) (ca. 800 basis functions) using 16 OpenMP threads of an Intel ${ }^{\circledR}$ Xeon ${ }^{\circledR}$ Gold $6148 \mathrm{CPU} @ 2.40 \mathrm{GHz}$. In comparison, the derivatives took twenty minutes for (1) and (3) and two minutes for (2) without DLU. The differences in isotropic hyperfine coupling constants predicted with and without DLU constitute less than $0.1 \%$ error introduced in all cases. On average, 10-20 SCF iterations were needed for the SO calculations based on converged UKS orbitals for x2c-TZVPPall-s/TPSS and x2c-TZVPPall-2c/TPSS, whereas approximately 40-60 SCF iterations were needed for x2c-TZVPPall-2c/PBE0 and x2c-QZVPall-2c/wB97X-D.

\section{Application to $\left[\mathrm{TbPc}_{2}\right]^{-}$with $S=3$}

So far, we have considered molecular systems with one unpaired electron, that is $S=1 / 2$. However, the Kramers-unrestricted non-collinear method is straightforwardly applicable to systems with more than one unpaired electron. ${ }^{24}$ To illustrate this feature of our implementation, we apply the mSNSO-DLU-X2C approach to $\left[\mathrm{TbPc}_{2}\right]^{-}$, a single molecule magnet with six unpaired electrons. This system is notable for its large hyperfine coupling arising from strong magnetic anisotropy along the easy axis. Furthermore, its valence structure differs significantly from the Ln complexes studied in Sec. 7, where the spin density arises purely from the $4 f$ shell as opposed to the $5 d / 6 s$ orbital. The molecular structure is depicted in Fig. 5. Note that the electronic ground-state of $\left[\mathrm{TbPc}_{2}\right]^{-}$is well separated from the excited states. 5

To study the HFC constant, we use the same methods as in the previous section.

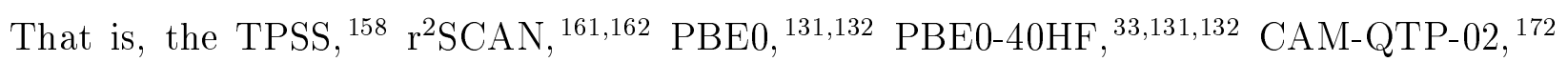
HSE06, $\frac{173-175}{175}$ and the $\omega$ B97X-D ${ }^{177}$ functionals are employed. The $\mathrm{x} 2 \mathrm{c}-\mathrm{TZVPPall}-2 \mathrm{c}$ basis set is used for $\mathrm{Tb}$ and the $\mathrm{x} 2 \mathrm{c}-\mathrm{SVP}$ all-2c basis set for all other atoms. ${ }^{106}$ This basis set setup 
Figure 5: Molecular structures of $\left[\mathrm{TbPc}_{2}\right]^{-}$. Pc denotes bis(phthalocyaninato). $\mathrm{H}$ atoms are omitted for clarity. Colors: Tb green, N blue, C grey.

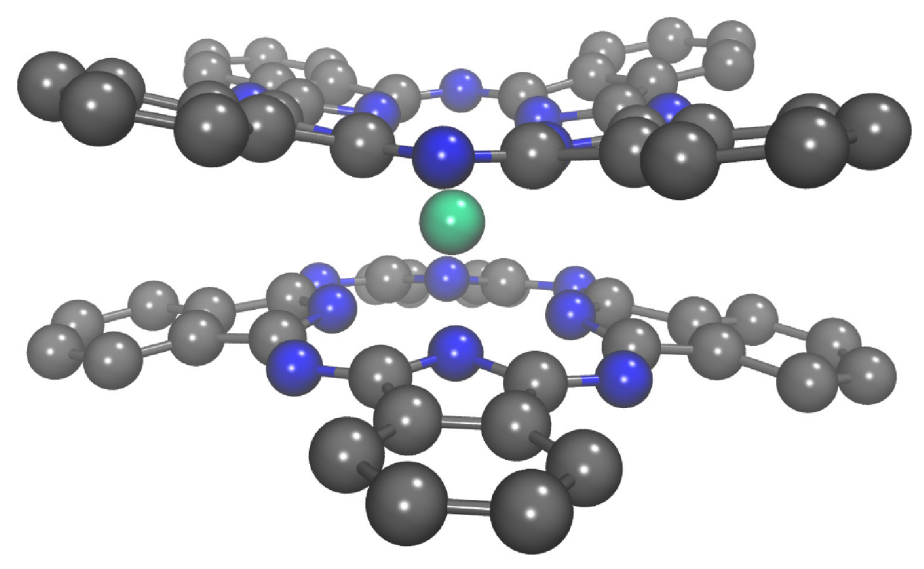

is motivated by the results observed in the last section. The isotropic hyperfine coupling constants calculated at the DFT level are given in Tab. 5.

Generally, the scalar-relativistic ansatz is not able to deliver accurate results, as it considers only the Fermi-contact and the spin-dipole contribution. For systems such as $\left[\mathrm{TbPc}_{2}\right]^{-}$ with $S=3$, the paramagnetic spin-orbit term is the leading contribution, and the Fermi contact term is minimal due to the primarily $4 f$ valence structure. ${ }^{5}$ Therefore, our SR results are almost universally off by a few hundred $\mathrm{MHz}$, and the error is greater than that observed for the La and Lu complexes in Sec. 7. In comparison, the two-component calculations result in good agreement with the experimental findings due to inclusion of this spin-orbit term. Similar to the previous sections, PBE0-HF and $\omega$ B97X-D perform remarkably well. In contrast, CAM-QTP-02 and HSE06 loose some ground for $\left[\mathrm{TbPc}_{2}\right]^{-} \cdot \mathrm{r}^{2} \mathrm{SCAN}$ is a notable exception for the scalar-relativistic results. This finding is likely caused by error cancellation as the PSO term and spin-orbit effects are pronounced for $\left[\mathrm{TbPc}_{2}\right]^{-}$in all calculations herein and in the literature.

The accuracy of our DFT-based X2C approach is on par with the multi-configurational ansatz by Wysocki and Park employing spin-orbit mean-field operators in scalar-relativistic low-order DKH. ${ }^{5}$ They obtained a HFC constant of ca. $6000 \mathrm{MHz}$ with an effective pseudospin Hamiltonian of $S=1 / 2$ and consequently mapping this for $\left[\mathrm{TbPc}_{2}\right]^{-}$with $S=3$ results in 
Table 5: Isotropic hyperfine coupling constant for $\left[\mathrm{TbPc}_{2}\right]^{-}$with the scalar-relativistic (SR) and the spin-orbit (SO) DLU-X2C Hamiltonian. The latter uses the mSNSO approximation. For brevity, only the basis set of the Tb is given here. The $\mathrm{x} 2 \mathrm{c}-\mathrm{SVPall}-2 \mathrm{c}$ basis set is employed for the light elements. Experimental results (Expt.) are taken from Refs. 226] and 227, see footnotes. All values are given in $\mathrm{MHz}$.

\begin{tabular}{llrc}
\hline Functional & Basis Set & \multicolumn{1}{c}{ SR } & SO \\
\hline TPSS & x2c-TZVPPall-2c & 189.2 & 352.4 \\
r $^{2}$ SCAN & x2c-TZVPPall-2c & 519.0 & 613.3 \\
PBE0 & x2c-TZVPPall-2c & 52.0 & 440.1 \\
PBE0-40HF & x2c-TZVPPall-2c & 26.4 & 510.5 \\
CAM-QTP-02 & x2c-TZVPPall-2c & -4.0 & 445.7 \\
HSE06 & x2c-TZVPPall-2c & 55.0 & 442.7 \\
$\omega B 97 X-D$ & x2c-TZVPPall-2c & 135.9 & 488.0 \\
\hline Expt. & & $519^{a}$ \\
Expt. & & $556^{a}$ \\
\hline${ }^{a}$ Result taken from Ref. 226 & \multicolumn{3}{c}{} \\
${ }^{b}$ Result taken from Ref. 227 &
\end{tabular}

$500 \mathrm{MHz}$. This shows that a sophisticated treatment of relativistic effects can be combined with DFT for lanthanide molecules beyond Kramers doublets if the electronic ground state is sufficiently separated from the excited states.

\section{Conclusions}

An efficient implementation of hyperfine coupling (HFC) matrix calculations in the quasirelativistic exact two-component (X2C) framework including the full spin-orbit $\mathrm{X} 2 \mathrm{C}$ derivative and the diagonal local approximation to the unitary decoupling transformation (DLU) has been reported. The method supports point and finite nucleus models for the scalar and vector potentials, is fully integral direct, and all integrals and algebraic manipulations are parallelized using OpenMP. The hyperfine coupling matrix can be computed in a "black-box" fashion in the non-collinear approach through incorporation of post-processing scripts into the TURBMOLE program suite.

Comparisons between the Dirac-Kohn-Sham (DKS) level and the X2C/DLU-X2C ansatz 
including the modified screened nuclear spin-orbit approximation (mSNSO) were carried out for 17 transition-metal compounds. The X2C calculations reproduce the DKS results well in each case for lower formal cost, and is further accelerated by the DLU scheme, which was found to introduce errors of just ca. $0.1 \mathrm{MHz}$. Inclusion of the full X2C Hamiltonian derivative led to corrections in the range of $1-15 \mathrm{MHz}$ in the HFC, which did not always bring the result closer to that of the DKS method. The worsening is likely due to the loss of error cancellation from neglecting the $\boldsymbol{X}$ and $\boldsymbol{R}$ derivatives, and may be amended through rigorous treatment of two-electron picture-change effects. When used with DLU, these derivatives do not contribute significantly to computational overhead, which is dominated by the twoelectron integral calculations.

In addition, an extensive study of general parameters, such as relativistic basis set and density functional approximation (DFA) was conducted. The segmented-contracted x2cQZVPall-2c basis set was found to represent a balanced choice for both accuracy of hyperfine coupling constant and computational cost, performing similarly to its uncontracted version. Other segmented-contracted bases, such as the Sapporo and Jorge bases, expectedly performed better with increasing cardinal number, but were inferior to x2c-QZVPall-2c and all uncontracted basis sets studied. In line with prior observations, Hartree-Fock exchange was found to be critical for accurate calculations of hyperfine coupling constants for the 12 transition-metal complexes studied in the DFA analysis. With the exception of $\mathrm{r}^{2} \mathrm{SCAN}$, global hybrid and range-separated hybrid functionals performed better in general than pure functionals when compared with experiment. CAM-QTP-02 and $\omega$ B97X-D show the smallest deviations from the experimental findings among the range-separated hybrids. However, range-separated hybrid functionals were not necessarily better than global hybrids, and common functionals such as PBE0 and TPSSh (ranked 6 and 10 out of the 22 studied) can be used without a significant compromise to accuracy.

mSNSO-DLU-X2C calculations were also performed on the Pt complex $\left[\mathrm{Pt}\left(\mathrm{C}_{6} \mathrm{Cl}_{5}\right)_{4}\right]^{-}$, which echoed the necessity of including Hartree-Fock exchange in the density functional 
approximation used for accurate HFC matrix calculations. Furthermore, these calculations demonstrated the substantial speedup achieved by application of DLU on X2C Hamiltonian derivatives, reducing the computation time from 1851 minutes to 41.7 minutes for the $\mathrm{Pt}$ complex at the x2c-QZVPall-2c level (3003 contracted and 4720 primitive basis functions) using 24 threads on an Intel ${ }^{\circledR}$ Xeon ${ }^{\circledR}$ Gold 6212U CPU @ 2.40 GHz.

We then applied the method to compute the hyperfine coupling constants for a series of three recently discovered $\mathrm{Ln}(\mathrm{II})$ single molecule magnets $\left[\mathrm{La}(\mathrm{OAr})_{3}\right]^{1-},\left[\mathrm{Lu}\left(\mathrm{NR}_{2}\right)_{3}\right]^{1-}$, and $\left[\mathrm{Lu}(\mathrm{OAr})_{3}\right]^{1-}$ with magnetic structure conducive to facilitating clock transitions. The results drastically correct previously obtained isotropic hyperfine coupling constants using scalar-relativistic density functional theory and a non-relativistic Fermi-contact expression, bringing the error down from thousands to tens of $\mathrm{MHz}$ when compared with experimental results. As demonstrated in this study, the non-relativistic HFC operator is insufficient for heavy atoms with valence orbitals of low angular momentum and large spin density near the nucleus. As this specific property is largely responsible for the desirable magnetic structure of this series, our results reaffirm the observation that efficient, "fully" relativistic or quasirelativistic ansätze are necessary to accurately describe the magnetic structure of candidate molecular qubit systems. Notably, the segmented-contracted triple- $\zeta$ x2c-TZVPPall-2c basis sets can be used for the lanthanide atom without a significant compromise to accuracy. The PBE0-40HF, CAM-QTP-02, and $\omega$ B97X-D functionals also perform well for the three Ln(II) single molecule magnets. In contrast to the transition-metal complexes, the pure density functionals already yield very reasonable results for these molecules.

The general applicability of the Kramers-unrestricted non-collinear ansatz is finally demonstrated for $\left[\mathrm{TbPc}_{2}\right]^{-}$with six unpaired electrons. Here, the scalar-relativistic X2C Hamiltonian is clearly insufficient as the paramagnetic spin-orbit contribution is the leading term, and consequently a quasi-relativistic spin-orbit approach is needed for accurate results. The PBE0-40HF and $\omega$ B97X-D functionals lead to an isotropic hyperfine coupling constant of $508 \mathrm{MHz}$ and $484 \mathrm{MHz}$, respectively. This is in remarkably good agreement with the ex- 
perimentally obtained result of $519 \mathrm{MHz}, 226$ and our results are on par with existing multiconfigurational methods. $[$ [

As an outlook, the implementations of the HFC matrix and NMR shifts in the X2C framework can be used to calculate the EPR g-tensors according to 24228

$$
g_{u v}=\frac{\mathrm{d}^{2} E}{\mathrm{~d} B_{u} \mathrm{~d} S_{v}}=\frac{2 c}{\left\langle\tilde{S}_{v}\right\rangle} \frac{\mathrm{d} E\left(J_{v}, \vec{B}\right)}{\mathrm{d} B_{u}}
$$

with the external magnetic field $\vec{B}$. Formally, the g-tensor should be computed using the restricted magnetic balance (RMB) condition ${ }^{[73}$ and gauge-including atomic orbitals (GIAOs) .229230 A respective implementation can be achieved through taking the spin densities from the HFC routines and the one- and two-electron integrals from the X2C NMR shift routines, $\frac{53174,76}{76}$ resulting in a calculation resembling two-component geometry gradients. .52280103 The specific methodology and working equations will be elaborated on in future work.

\section{Acknowledgement}

Y.J.F. acknowledges financial support by TURBOMOLE GmbH and was further supported by a fellowship of Fonds der Chemischen Industrie (FCI, German Chemical Industry Funds). J.M.Y. acknowledges support from the National Science Foundation under grant No. DCE1839285. A portion of the calculations reported in this work was performed using the highperformance computing (HPC) infrastructure available at the Research Cyberinfrastructure Center (RCIC) at the University of California, Irvine (UCI) and supported by the National Science Foundation under grant No. CNS-1828779. The authors are grateful to Filipp Furche and Florian Weigend for fruitful discussions. We thank Ansgar Pausch for discussions on the SCF behavior and the implementation of the canonical orthogonalization to confirm the ground-state energy in critical cases with decontracted basis sets. Y.J.F. thanks Sebastian Gillhuber for helpful discussions. 


\section{Supporting Information Available}

The Supporting Information is available on the ACS Publications website at DOI: XX/xxxxx

- Comparison to ZORA results for $\mathrm{CH}_{3}, \mathrm{HCO}, \mathrm{HSiO}, \mathrm{HSiS}, \mathrm{SiOH}, \mathrm{SiSH}, \mathrm{HgH}, \mathrm{HgF}$, $\mathrm{HgCN}$, and $\mathrm{HgAg}(\mathrm{PDF})$

- Optimized structures of HCO, HSiO, HSiS, $\mathrm{SiOH}, \mathrm{SiSH}, \mathrm{HgH}, \mathrm{HgF}, \mathrm{HgCN}$, and HgAg at the BP86/x2c-TZVPall-2c level (PDF)

- Individual results for the basis set study in Sec. $5.2(\mathrm{PDF})$

- Individual results for the DFT study in Sec. 5.3 (PDF)

- Uncontracted x2c-type basis sets for Sec. 5.2, i.e. x2c-SVPall-2c-unc, x2c-TZVPall-2cunc, and x2c-QZVPall-2c-unc (txt)

\section{References}

(1) Wolfowicz, G.; Tyryshkin, A. M.; George, R. E.; Riemann, H.; Abrosimov, N. V.; Becker, P.; Pohl, H.-J.; Thewalt, M. L. W.; Lyon, S. A.; Morton, J. L. Atomic clock transitions in silicon-based spin qubits. Nat. Nanotechnol. 2013, 8, 561-564.

(2) Shiddiq, M.; Komijani, D.; Duan, Y.; Gaita-Ariño,; Coronado, E.; Hill, S. Enhancing coherence in molecular spin qubits via atomic clock transitions. Nature 2016, 531, $348-351$.

(3) Zadrozny, J. M.; Niklas, J.; Poluektov, O. G.; Freedman, D. E. Millisecond Coherence Time in a Tunable Molecular Electronic Spin Qubit. ACS. Cent. Sci. 2015, 1, 488-492.

(4) Lan, T. N.; Kurashige, Y.; Yanai, T. Toward Reliable Prediction of Hyperfine Coupling Constants Using Ab Initio Density Matrix Renormalization Group Method: Diatomic

${ }^{2} \Sigma$ and Vinyl Radicals as Test Cases. J. Chem. Theory Comput. 2014, 10, 1953-1967. 
(5) Wysocki, A. L.; Park, K. Nature of Hyperfine Interactions in $\mathrm{TbPc}_{2}$ Single-Molecule Magnets: Multiconfigurational Ab Initio Study. Inorg. Chem. 2020, 59, 2771-2780.

(6) Hermosilla, L.; Calle, P.; García de la Vega, J. M.; Sieiro, C. Density Functional Theory Predictions of Isotropic Hyperfine Coupling Constants. J. Phys. Chem. A 2005, 109, $1114-1124$.

(7) Datta, D.; Gauss, J. Accurate Prediction of Hyperfine Coupling Tensors for Main Group Elements Using a Unitary Group Based Rigorously Spin-Adapted CoupledCluster Theory. J. Chem. Theory Conput. 2019, 15, 1572-1592.

(8) Kossmann, S.; Neese, F. Correlated ab Initio Spin Densities for Larger Molecules: Orbital-Optimized Spin- Component-Scaled MP2 Method. J. Phys. Chem. A 2010, $114,11768-11781$.

(9) Pyykkö, P. Relativistic effects in structural chemistry. Chem. Rev. 1988, 88, 563-594.

(10) Dyall, K. G. Interfacing relativistic and nonrelativistic methods. IV. One- and twoelectron scalar approximations. J. Chem. Phys. 2001, 115, 9136-9143.

(11) Sharkas, K.; Pritchard, B.; Autschbach, J. Effects from Spin-Orbit Coupling on Electro-Nucleus Hyperfine Coupling Calculated at the Restricted Active Space Level for Kramers Doublets. J. Chem. Theory Comput. 2015, 11, 538-549.

(12) Verma, P.; Autschbach, J. Relativistic Density Functional Calculations of Hyperfine Coupling with Variational versus Perturbational Treatment of Spin-Orbit Coupling. J. Chem. Theory Comput. 2013, 9, 1932-1948.

(13) Kaupp, M., Bühl, M., Malkin, V. G., Eds. Calculation of NMR and EPR Parameters. Theory and Applications; Wiley-VCH: Weinheim, Germany, 2004.

(14) Autschbach, J. Relativistic Effects on Magnetic Resonance Parameters and Other Properties of Inorganic Molecules and Metal Complexes. In Relativistic Methods for 
Chemists; Barysz, M., Ishikawa, Y., Eds.; Springer Netherlands: Dordrecht, 2010; pp $521-598$.

(15) Autschbach, J. Perspective: Relativistic effects. J. Chem. Phys. 2012, 136, 150902.

(16) Autschbach, J. Relativistic calculations of magnetic resonance parameters: background and some recent developments. Philos. Trans. A Math. Phys. Eng. Sci. 2014, 372, 20120489.

(17) Saue, T. Relativistic Hamiltonians for Chemistry: A Primer. ChemPhysChem 2011, 12, 3077-3094.

(18) Pyykkö, P. Relativistic Effects in Chemistry: More Common Than You Thought. Annu. Rev. Phys. Chem. 2012, 63, 45.

(19) Dyall, K. G.; Fægri Jr., K. Introduction to Relativistic Quantum Chemistry; Oxford University Press: New York, 2007.

(20) Reiher, M.; Wolf., A. Relativistic Quantum Chemistry - The Fundamental Theory of Molecular Science, 2nd ed.; Wiley-VCH: Weinheim, Germany, 2015.

(21) Liu, W., Ed. Handbook of Relativistic Quantum Chemistry; Springer: Berlin, Heidelberg, 2017.

(22) Kundu, K.; White, J. R. K.; Moehring, S. A.; Yu, J. M.; Ziller, J. W.; Furche, F.; Evans, W. J.; Hill, S. Clock Transition Due to a Record 1240 G Hyperfine Interaction in a Lu(II) Molecular Spin Qubit. ChemRxiv 2021, DOI: 10.26434/chemrxiv.14399333.v1.

(23) Smith, R. L.; Wysocki, A. L.; Park, K. Electrically tuned hyperfine spectrum in neutral $\mathrm{Tb}(\mathrm{II})\left(\mathrm{Cp}^{\mathrm{iPr} 5}\right)_{2}$ single-molecule magnet. Phys. Chem. Chem. Phys. 2020, 22, 2179321800. 
(24) Malkin, I.; Malkina, O. L.; Malkin, V. G.; Kaupp, M. Relativistic two-component calculations of electronic g-tensors that include spin polarization. J. Chem. Phys. 2005, 123, 244103.

(25) Douglas, M.; Kroll, N. M. Quantum electrodynamical corrections to the fine structure of helium. Ann. Phys. (NY) 1974, 82, 89-155.

(26) Hess, B. A. Relativistic electronic-structure calculations employing a two-component no-pair formalism with external-field projection operators. Phys. Rev. A 1986, 33, $3742-3748$.

(27) Jansen, G.; Hess, B. A. Revision of the Douglas-Kroll transformation. Phys. Rev. A 1989, 39, 6016-6017.

(28) Chang, C.; Pelissier, M.; Durand, P. Regular Two-Component Pauli-Like Effective Hamiltonians in Dirac Theory. Phys. Scr. 1986, 34, 394-404.

(29) van Lenthe, E.; Baerends, E. J.; Snijders, J. G. Relativistic regular two-component Hamiltonians. J. Phys. Chem. 1993, 99, 4597-4610.

(30) van Lenthe, E.; Baerends, E. J.; Snijders, J. G. Relativistic total energy using regular approximations. J. Chem. Phys. 1994, 101, 9783-9792.

(31) Autschbach, J.; Patchkovskii, S.; Pritchard, B. Calculation of Hyperfine Tensors and Paramagnetic NMR Shifts Using the Relativistic Zeroth-Order Regular Approximation and Density Functional Theory. J. Chem. Theory Comput. 2011, 7, 2175-2188.

(32) Malkin, E.; Repiský, M.; Komorovský, S.; Mach, P.; Malkina, O. L.; Malkin, V. G. Effects of finite size nuclei in relativistic four-component calculations of hyperfine structure. J. Chem. Phys. 2011, 134, 044111.

(33) Gohr, S.; Hrobárik, P.; Repiský, M.; Komorovský, S.; Ruud, K.; Kaupp, M. FourComponent Relativistic Density Functional Theory Calculations of EPR g- and 
Hyperfine-Coupling Tensors Using Hybrid Functionals: Validation on TransitionMetal Complexes with Large Tensor Anisotropies and Higher-Order Spin-Orbit Effects. J. Phys. Chem. A 2015, 119, 12892-12905.

(34) Kutzelnigg, W.; Liu, W. Quasirelativistic theory equivalent to fully relativistic theory. J. Chem. Phys. 2005, 123, 241102.

(35) Liu, W.; Kutzelnigg, W. Quasirelativistic theory. II. Theory at matrix level. J. Chem. Phys. 2007, 126, 114107.

(36) Liu, W.; Peng, D. Infinite-order quasirelativistic density functional method based on the exact matrix quasirelativistic theory. J. Chem. Phys. 2006, 125, 044102.

(37) Liu, W.; Peng, D. Erratum: "Infinite-order quasirelativistic density functional method based on the exact matrix quasirelativistic theory" [J. Chem. Phys. 125, 044102 (2006)]. J. Chem. Phys. 2006, 125, 149901.

(38) Iliaš, M.; Saue, T. An infinite-order two-component relativistic Hamiltonian by a simple one-step transformation. J. Chem. Phys. 2007, 126, 064102.

(39) Liu, W.; Peng, D. Exact two-component Hamiltonians revisited. J. Chem. Phys. 2009, $131,031104$.

(40) Peng, D.; Liu, W.; Xiao, Y.; Cheng, L. Making four- and two-component relativistic density functional methods fully equivalent based on the idea of "from atoms to molecule". J. Chem. Phys. 2007, 127, 104106.

(41) Autschbach, J. Relativistic Effects on Electron-Nucleus Hyperfine Coupling Studied with an Exact 2-Component (X2C) Hamiltonian. J. Chem. Theory Comput. 2017, 13, $710-718$. 
(42) Feng, R.; Duignan, T. J.; Autschbach, J. Electron-Nucleus Hyperfine Coupling Calculated from Restricted Active Space Wavefunctions and an Exact Two-Component Hamiltonian. J. Chem. Theory Comput. 2021, 17, 255-268.

(43) Wodyński, A.; Kaupp, M. Density Functional Calculations of EPR g- and HyperfineCoupling Tensors Using the Exact Two-Component (X2C) Transformation and Efficient Approximations to the Two-Electron Spin-Orbit Terms. J. Phys. Chem. A 2019, 123, 5660-5672.

(44) Liu, W. Ideas of relativistic quantum chemistry. Mol. Phys. 2010, 108, 1679-1706.

(45) Peng, D.; Reiher, M. Exact decoupling of the relativistic Fock operator. Theor. Chem. Acc. 2012, 131, 1081.

(46) Cheng, L.; Gauss, J. Analytic energy gradients for the spin-free exact two-component theory using an exact block diagonalization for the one-electron Dirac Hamiltonian. J. Chem. Phys. 2011, 135, 084114.

(47) Cheng, L.; Stopkowicz, S.; Gauss, J. Analytic energy derivatives in relativistic quantum chemistry. Int. J. Quantum Chem. 2014, 114, 1108-1127.

(48) Zou, W.; Filatov, M.; Cremer, D. Development and application of the analytical energy gradient for the normalized elimination of the small component method. J. Chem. Phys. 2011, 134, 244117.

(49) Filatov, M.; Zou, W.; Cremer, D. Analytic Calculation of Isotropic Hyperfine Structure Constants Using the Normalized Elimination of the Small Component Formalism. J. Phys. Chem. A 2012, 116, 3481-3486.

(50) Cremer, D.; Zou, W.; Filatov, M. Dirac-exact relativistic methods: the normalized elimination of the small component method. Wiley Interdiscip. Rev.: Comput. Mol. Sci. 2014, 4, 436-467. 
(51) Peng, D.; Middendorf, N.; Weigend, F.; Reiher, M. An efficient implementation of two-component relativistic exact-decoupling methods for large molecules. J. Chem. Phys. 2013, 138, 184105.

(52) Franzke, Y. J.; Middendorf, N.; Weigend, F. Efficient implementation of one- and two-component analytical energy gradients in exact two-component theory. J. Chem. Phys. 2018, 148, 104410.

(53) Franzke, Y. J.; Weigend, F. NMR Shielding Tensors and Chemical Shifts in ScalarRelativistic Local Exact Two-Component Theory. J. Chem. Theory Comput. 2019, $15,1028-1043$.

(54) Kehry, M.; Franzke, Y. J.; Holzer, C.; Klopper, W. Quasirelativistic two-component core excitations and polarisabilities from a damped-response formulation of the BetheSalpeter equation. Mol. Phys. 2020, 118, e1755064.

(55) Franzke, Y. J.; Mack, F.; Weigend, F. NMR Indirect Spin-Spin Coupling Constants in a Modern Quasirelativistic Density Functional Framework. J. Chem. Theory Comput. 2021, 17, 3974-3994.

(56) Schmitt, S.; Jost, P.; van Wüllen, C. Zero-field splittings from density functional calculations: Analysis and improvement of known methods. J. Chem. Phys. 2011, $134,194113$.

(57) Verma, P.; Autschbach, J. Variational versus Perturbational Treatment of Spin-Orbit Coupling in Relativistic Density Functional Calculations of Electronic g Factors: Effects from Spin-Polarization and Exact Exchange. J. Chem. Theory Comput. 2013, 9, $1052-1067$.

(58) Remigio, R. D.; Repisky, M.; Komorovsky, S.; Hrobarik, P.; Frediani, L.; Ruud, K. Four-component relativistic density functional theory with the polarisable continuum 
model: application to EPR parameters and paramagnetic NMR shifts. Mol. Phys. 2017, 115, 214-227.

(59) Yoshizawa, T. On the development of the exact two-component relativistic method for calculating indirect NMR spin-spin coupling constants. Chem. Phys. 2019, 518, $112-122$.

(60) Foldy, L. L.; Wouthuysen, S. A. On the Dirac Theory of Spin 1/2 Particles and Its Non-Relativistic Limit. Phys. Rev. 1950, 78, 29-36.

(61) Gell-Mann, M. The interpretation of the new particles as displaced charge multiplets. Nuovo Cim. 1956, 4, 848-866.

(62) Bohr, A.; Weisskopf, V. F. The Influence of Nuclear Structure on the Hyperfine Structure of Heavy Elements. Phys. Rev. 1950, 77, 94-98.

(63) Hennum, A. C.; Klopper, W.; Helgaker, T. Direct perturbation theory of magnetic properties and relativistic corrections for the point nuclear and Gaussian nuclear models. J. Chem. Phys. 2001, 115, 7356-7363.

(64) Visscher, L.; Dyall, K. G. Dirac-Fock atomic electronic structure calculations using different nuclear charge distributions. At. Data Nucl. Data Tables 1997, 67, 207-224.

(65) Stanton, R. E.; Havriliak, S. Kinetic balance: A partial solution to the problem of variational safety in Dirac calculations. J. Chem. Phys. 1984, 81, 1910-1918.

(66) Visser, O.; Aerts, P.; Hegarty, D.; Nieuwpoort, W. The use of gaussian nuclear charge distributions for the calculation of relativistic electronic wavefunctions using basis set expansions. Chem. Phys. Lett. 1987, 134, 34-38.

(67) Ishikawa, Y.; Baretty, R.; Binning, R. Relativistic Gaussian basis set calculations on one-electron ions with a nucleus of finite extent. Chem. Phys. Lett. 1985, 121, 130-133. 
(68) Repiský, M.; Komorovský, S.; Malkina, O. L.; Malkin, V. G. Restricted magnetically balanced basis applied for relativistic calculations of indirect nuclear spin-spin coupling tensors in the matrix Dirac-Kohn-Sham framework. Chem. Phys. 2009, 356, $236-242$.

(69) Enevoldsen, T.; Visscher, L.; Saue, T.; Jensen, H. J. A.; Oddershede, J. Relativistic four-component calculations of indirect nuclear spin-spin couplings in $\mathrm{MH}_{4}(\mathrm{M}=\mathrm{C}, \mathrm{Si}$, Ge, Sn, $\mathrm{Pb})$ and $\mathrm{Pb}\left(\mathrm{CH}_{3}\right)_{3} \mathrm{H}$. J. Chem. Phys. 2000, 112, 3493-3498.

(70) Visscher, L.; Enevoldsen, T.; Saue, T.; Jensen, H. J. A.; Oddershede, J. Full fourcomponent relativistic calculations of NMR shielding and indirect spin-spin coupling tensors in hydrogen halides. J. Comput. Chem. 1999, 20, 1262-1273.

(71) Gomez, S. S.; Romero, R. H.; Aucar, G. A. Fully relativistic calculation of nuclear magnetic shieldings and indirect nuclear spin-spin couplings in group-15 and -16 hydrides. J. Chem. Phys. 2002, 117, 7942-7946.

(72) Giménez, C. A.; Maldonado, A. F.; Aucar, G. A. Relativistic and electron correlation effects on NMR J-coupling of $\mathrm{Sn}$ and $\mathrm{Pb}$ containing molecules. Theor. Chem. Acc. 2016, 135, 201.

(73) Komorovský, S.; Repiský, M.; Malkina, O. L.; Malkin, V. G.; Malkin Ondík, I.; Kaupp, M. A fully relativistic method for calculation of nuclear magnetic shielding tensors with a restricted magnetically balanced basis in the framework of the matrix Dirac-Kohn-Sham equation. J. Chem. Phys. 2008, 128, 104101.

(74) Cheng, L.; Gauss, J.; Stanton, J. F. Treatment of scalar-relativistic effects on nuclear magnetic shieldings using a spin-free exact-two-component approach. J. Chem. Phys. 2013, 139, 054105.

(75) Yoshizawa, T.; Zou, W.; Cremer, D. Calculations of atomic magnetic nuclear shielding 
constants based on the two-component normalized elimination of the small component method. J. Chem. Phys. 2017, 146, 134109.

(76) Yoshizawa, T.; Hada, M. Calculations of nuclear magnetic shielding constants based on the exact two-component relativistic method. J. Chem. Phys. 2017, 147, 154104.

(77) Sun, S.; Li, X. Relativistic Effects in Magnetic Circular Dichroism: Restricted Magnetic Balance and Temperature Dependence. J. Chem. Theory Comput. 2020, 16, 4533-4542.

(78) Boettger, J. C. Approximate two-electron spin-orbit coupling term for densityfunctional-theory DFT calculations using the Douglas-Kroll-Hess transformation. Phys. Rev. B 2000, 62, 7809-7815.

(79) Filatov, M.; Zou, W.; Cremer, D. Spin-orbit coupling calculations with the twocomponent normalized elimination of the small component method. J. Chem. Phys. 2013, 139, 014106.

(80) Zou, W.; Filatov, M.; Cremer, D. Analytical energy gradient for the two-component normalized elimination of the small component method. J. Chem. Phys. 2015, 142, 214106.

(81) Yoshizawa, T.; Zou, W.; Cremer, D. Calculations of electric dipole moments and static dipole polarizabilities based on the two-component normalized elimination of the small component method. J. Chem. Phys. 2016, 145, 184104.

(82) van Wüllen, C.; Michauk, C. Accurate and efficient treatment of two-electron contributions in quasirelativistic high-order Douglas-Kroll density-functional calculations. J. Chem. Phys. 2005, 123, 204113.

(83) Dyall, K. G. Interfacing relativistic and nonrelativistic methods. I. Normalized elimi- 
nation of the small component in the modified Dirac equation. J. Chem. Phys. 1997, $106,9618-9626$.

(84) Dyall, K. G. Interfacing relativistic and nonrelativistic methods. II. Investigation of a low-order approximation. J. Chem. Phys. 1998, 109, 4201-4208.

(85) Dyall, K. G.; Enevoldsen, T. Interfacing relativistic and nonrelativistic methods. III. Atomic 4-spinor expansions and integral approximations. J. Chem. Phys. 1999, 111, 10000-10007.

(86) Zou, W.; Filatov, M.; Cremer, D. Development, Implementation, and Application of an Analytic Second Derivative Formalism for the Normalized Elimination of the Small Component Method. J. Chem. Theory Comput. 2012, 8, 2617-2629.

(87) Kutzelnigg, W. Origin and meaning of the Fermi contact interaction. Theor. Chim. Acta 1988, 73, 173-200.

(88) Stevens, R. M.; Pitzer, R. M.; Lipscomb, W. N. Perturbed Hartree-Fock Calculations. I. Magnetic Susceptibility and Shielding in the LiH Molecule. J. Chem. Phys. 1963, $38,550-560$.

(89) O'Shea, S. F.; Santry, D. P. Non-empirical molecular orbital theory of the electronic structure of molecular crystals. Theor. Chim. Acta 1975, 37, 1-16.

(90) Lawson, C. L.; Hanson, R. J.; Kincaid, D. R.; Krogh, F. T. Basic Linear Algebra Subprograms for Fortran Usage. ACM Trans. Math. Softw. 1979, 5, 308-323.

(91) Blackford, L. S.; Demmel, J.; Dongarra, J.; Duff, I.; Hammarling, S.; Henry, G.; Heroux, M.; Kaufman, L.; Lumsdaine, A.; Petitet, A.; Pozo, R.; Remington, K.; Whaley, R. C. An updated set of basic linear algebra subprograms (BLAS). ACM Trans. Math. Softw. 2002, 28, 135-151. 
(92) Ahlrichs, R.; Bär, M.; Häser, M.; Horn, H.; Kölmel, C. Electronic structure calculations on workstation computers: The program system turbomole. Chem. Phys. Lett. 1989, 162, 165-169.

(93) Furche, F.; Ahlrichs, R.; Hättig, C.; Klopper, W.; Sierka, M.; Weigend, F. Turbomole. Wiley Interdiscip. Rev.: Comput. Mol. Sci. 2014, 4, 91-100.

(94) Balasubramani, S. G.; Chen, G. P.; Coriani, S.; Diedenhofen, M.; Frank, M. S.; Franzke, Y. J.; Furche, F.; Grotjahn, R.; Harding, M. E.; Hättig, C.; Hellweg, A.; Helmich-Paris, B.; Holzer, C.; Huniar, U.; Kaupp, M.; Marefat Khah, A.; Karbalaei Khani, S.; Müller, T.; Mack, F.; Nguyen, B. D.; Parker, S. M.; Perlt, E.; Rappoport, D.; Reiter, K.; Roy, S.; Rückert, M.; Schmitz, G.; Sierka, M.; Tapavicza, E.; Tew, D. P.; van Wüllen, C.; Voora, V. K.; Weigend, F.; Wodyński, A.; Yu, J. M. TURBOMOLE: Modular program suite for ab initio quantum-chemical and condensedmatter simulations. J. Chem. Phys. 2020, 152, 184107.

(95) Developers' version of TURBOMOLE V7.5.1 2021, a development of University of Karlsruhe and Forschungszentrum Karlsruhe GmbH, 1989-2007, TURBOMOLE GmbH, since 2007; available from https://www.turbomole.org (retrieved August 28, 2021).

(96) Dupuis, M.; Rys, J.; King, H. F. Evaluation of molecular integrals over Gaussian basis functions. J. Chem. Phys. 1976, 65, 111-116.

(97) Rys, J.; Dupuis, M.; King, H. F. Computation of electron repulsion integrals using the rys quadrature method. J. Comput. Chem. 1983, 4, 154-157.

(98) Lide, D. R., Ed. CRC Handbook of Chemistry and Physics, 78th ed.; CRC Press: Boca Ratón, USA, 1997.

(99) Stone, N. Table of nuclear magnetic dipole and electric quadrupole moments. At. Data Nucl. Data Tables 2005, 90, 75-176. 
(100) Mack, F.; Schattenberg, C. J.; Kaupp, M.; Weigend, F. Nuclear Spin-Spin Couplings: Efficient Evaluation of Exact Exchange and Extension to Local Hybrid Functionals. J. Phys. Chem. A 2020, 124, 8529-8539.

(101) CODATA internationally recommened 2018 values of the fundamental physical constans, https://physics.nist.gov/cuu/Constants/index.html (retrieved December 28, 2020).

(102) Armbruster, M. K.; Weigend, F.; van Wüllen, C.; Klopper, W. Self-consistent treatment of spin-orbit interactions with efficient Hartree-Fock and density functional methods. Phys. Chem. Chem. Phys. 2008, 10, 1748-1756.

(103) Baldes, A.; Weigend, F. Efficient two-component self-consistent field procedures and gradients: implementation in TURBOMOLE and application to $\mathrm{Au}_{20}^{-}$. Mol. Phys. 2013, 111, 2617-2624.

(104) Plessow, P.; Weigend, F. Seminumerical calculation of the Hartree-Fock exchange matrix: Application to two-component procedures and efficient evaluation of local hybrid density functionals. J. Comput. Chem. 2012, 33, 810-816.

(105) Holzer, C. An improved seminumerical Coulomb and exchange algorithm for properties and excited states in modern density functional theory. J. Chem. Phys. 2020, 153, 184115.

(106) Pollak, P.; Weigend, F. Segmented Contracted Error-Consistent Basis Sets of Doubleand Triple- $\zeta$ Valence Quality for One- and Two-Component Relativistic All-Electron Calculations. J. Chem. Theory Comput. 2017, 13, 3696-3705.

(107) OpenMP Architecture Review Boards, OpenMP API shared-memory parallel programming. https: //www . openmp.org (retrieved September 26, 2021). 
(108) Holzer, C.; Franzke, Y. J. OpenMP version of ridft, rdgrad, and egrad with contributions to mpshift, dscf, and grad; improved OpenMP version of aoforce and escf, released with TURBOMOLE V7.4 and further improved in TURBOMOLE V7.5.

(109) Eichkorn, K.; Treutler, O.; Öhm, H.; Häser, M.; Ahlrichs, R. Auxiliary Basis Sets to Approximate Coulomb Potentials. Chem. Phys. Lett. 1995, 242, 283-290.

(110) Weigend, F.; Kattannek, M.; Ahlrichs, R. Approximated electron repulsion integrals: Cholesky decomposition versus resolution of the identity methods. J. Chem. Phys. 2009, 130, 164106.

(111) Sierka, M.; Hogekamp, A.; Ahlrichs, R. Fast evaluation of the Coulomb potential for electron densities using multipole accelerated resolution of identity approximation. $J$. Chem. Phys. 2003, 118, 9136-9148.

(112) Weigend, F. A fully direct RI-HF algorithm: Implementation, optimised auxiliary basis sets, demonstration of accuracy and efficiency. Phys. Chem. Chem. Phys. 2002, 4, 4285-4291.

(113) Klamt, A.; Schüürmann, G. COSMO: a new approach to dielectric screening in solvents with explicit expressions for the screening energy and its gradient. J. Chem. Soc., Perkin Trans. 2 1993, 799-805.

(114) Schäfer, A.; Klamt, A.; Sattel, D.; Lohrenz, J. C. W.; Eckert, F. COSMO Implementation in TURBOMOLE: Extension of an efficient quantum chemical code towards liquid systems. Phys. Chem. Chem. Phys. 2000, 2, 2187-2193.

(115) Franzke, Y. J.; Treß, R.; Pazdera, T. M.; Weigend, F. Error-consistent segmented contracted all-electron relativistic basis sets of double- and triple-zeta quality for NMR shielding constants. Phys. Chem. Chem. Phys. 2019, 21, 16658-16664. 
(116) Perdew, J. P.; Schmidt, K. Jacob's ladder of density functional approximations for the exchange-correlation energy. AIP Conf. Proc. 2001, 577, 1-20.

(117) Becke, A. D. Perspective: Fifty years of density-functional theory in chemical physics. J. Chem. Phys. 2014, 140, 18A301.

(118) Mardirossian, N.; Head-Gordon, M. Thirty years of density functional theory in computational chemistry: an overview and extensive assessment of 200 density functionals. Mol. Phys. 2017, 115, 2315-2372.

(119) Marques, M. A. L.; Oliveira, M. J. T.; Burnus, T. Libxc: A library of exchange and correlation functionals for density functional theory. Comput. Phys. Commun. 2012, $183,2272-2281$.

(120) Lehtola, S.; Steigemann, C.; Oliveira, M. J. T.; Marques, M. A. L. Recent developments in libxc - A comprehensive library of functionals for density functional theory. SoftwareX 2018, \%, 1-5.

(121) Libxc. 5.1.4, available from https://www.tddft.org/programs/libxc/ (retrieved July 30, 2021).

(122) Ekström, U.; Visscher, L.; Bast, R.; Thorvaldsen, A. J.; Ruud, K. Arbitrary-Order Density Functional Response Theory from Automatic Differentiation. J. Chem. Theory Comput. 2010, 6, 1971-1980.

(123) Gillhuber, S.; Franzke, Y. J.; Weigend, F. Paramagnetic NMR Shielding Tensors and Ring Currents: Efficient Implementation and Application to Heavy Element Compounds. J. Phys. Chem. A 2021, 125, 9707-9723.

(124) Repisky, M.; Komorovsky, S.; Kadek, M.; Konecny, L.; Ekström, U.; Malkin, E.; Kaupp, M.; Ruud, K.; Malkina, O. L.; Malkin, V. G. ReSpect: Relativistic spectroscopy DFT program package. J. Chem. Phys. 2020, 152, 184101. 
(125) Tutorial for EPR A tensors with the ReSpect program suite, http://www. respectprogram.org/tutorials-hfcc.html (retrieved August 17, 2021).

(126) Kutzelnigg, W.; Fleischer, U.; Schindler, M. The IGLO-Method: Ab-initio Calculation and Interpretation of NMR Chemical Shifts and Magnetic Susceptibilities. In Deuterium and Shift Calculation; Springer: Berlin, Heidelberg, Germany, 1991; pp $165-262$.

(127) Dyall, K. G. Relativistic Quadruple-Zeta and Revised Triple-Zeta and Double-Zeta Basis Sets for the 4p, 5p, and 6p Elements. Theor. Chem. Acc. 2006, 115, 441-447.

(128) Basis sets available from the Dirac program web site, http://dirac.chem.sdu.dk (retrieved March 26, 2020).

(129) Dyall, K. G. Relativistic double-zeta, triple-zeta, and quadruple-zeta basis sets for the 4d elements Y-Cd. Theor. Chem. Acc. 2007, 117, 483-489.

(130) Gomes, A. S. P.; Dyall, K. G.; Visscher, L. Relativistic double-zeta, triple-zeta, and quadruple-zeta basis sets for the lanthanides La-Lu. Theor. Chem. Acc. 2010, 127, $369-381$.

(131) Perdew, J. P.; Burke, K.; Ernzerhof, M. Generalized Gradient Approximation Made Simple. Phys. Rev. Lett. 1996, r7, 3865-3868.

(132) Adamo, C.; Barone, V. Toward reliable density functional methods without adjustable parameters: The PBE0 model. J. Chem. Phys. 1999, 110, 6158-6170.

(133) Treutler, O.; Ahlrichs, R. Efficient molecular numerical integration schemes. J. Chem. Phys. 1995, 102, 346-354.

(134) Treutler, O. Entwicklung und Anwendung von Dichtefunktionalmethoden. Ph.D. thesis, University of Karlsruhe (TH), Germany, 1995. 
(135) Jensen, F. The Basis Set Convergence of Spin-Spin Coupling Constants Calculated by Density Functional Methods. J. Chem. Theory Comput. 2006, 2, 1360-1369.

(136) Dunning, T. H. Gaussian basis sets for use in correlated molecular calculations. I. The atoms boron through neon and hydrogen. J. Chem. Phys. 1989, 90, 1007-1023.

(137) Woon, D. E.; Dunning, T. H. Gaussian basis sets for use in correlated molecular calculations. III. The atoms aluminum through argon. J. Chem. Phys. 1993, 98, 13581371.

(138) Benedikt, U.; Auer, A. A.; Jensen, F. Optimization of augmentation functions for correlated calculations of spin-spin coupling constants and related properties. J. Chem. Phys. 2008, 129, 064111.

(139) Noro, T.; Sekiya, M.; Koga, T. Segmented contracted basis sets for atoms H through Xe: Sapporo-(DK)-nZP sets (n = D, T, Q). Theor. Chem. Acc. 2012, 131, 1124.

(140) Noro, T.; Sekiya, M.; Koga, T. Sapporo-(DKH3)-nZP (n = D, T, Q) sets for the sixth period s-, d-, and p-block atoms. Theor. Chem. Acc. 2013, 132, 1363.

(141) Jorge, F. E.; Canal Neto, A.; Camiletti, G. G.; Machado, S. F. Contracted Gaussian basis sets for Douglas-Kroll-Hess calculations: Estimating scalar relativistic effects of some atomic and molecular properties. J. Chem. Phys. 2009, 130, 064108.

(142) Campos, C. T.; Jorge, F. E. Triple zeta quality basis sets for atoms Rb through Xe: application in $\operatorname{CCSD}(\mathrm{T})$ atomic and molecular property calculations. Mol. Phys. 2013, 111, 167-173.

(143) Martins, L.; Jorge, F.; Machado, S. All-electron segmented contraction basis sets of triple zeta valence quality for the fifth-row elements. Mol. Phys. 2015, 113, 3578-3586. 
(144) Franzke, Y. J.; Spiske, L.; Pollak, P.; Weigend, F. Segmented Contracted ErrorConsistent Basis Sets of Quadruple- $\zeta$ Valence Quality for One- and Two-Component Relativistic All-Electron Calculations. J. Chem. Theory Comput. 2020, 16, 5658-5674.

(145) Zobel, J. P.; Widmark, P.-O.; Veryazov, V. The ANO-R Basis Set. J. Chem. Theory Comput. 2020, 16, 278-294.

(146) Roos, B. O.; Lindh, R.; Malmqvist, P.-A.; Veryazov, V.; Widmark, P.-O. Main Group Atoms and Dimers Studied with a New Relativistic ANO Basis Set. J. Phys. Chem. A 2004, 108, 2851-2858.

(147) Roos, B. O.; Lindh, R.; Malmqvist, P.-A.; Veryazov, V.; Widmark, P.-O. New Relativistic ANO Basis Sets for Transition Metal Atoms. J. Phys. Chem. A 2005, 109, $6575-6579$.

(148) Pritchard, B. P.; Altarawy, D.; Didier, B.; Gibson, T. D.; Windus, T. L. New Basis Set Exchange: An Open, Up-to-Date Resource for the Molecular Sciences Community. J. Chem. Inf. Model. 2019, 59, 4814-4820.

(149) Basis set exchange library ver2, BSE Library v0.8.12, https://www. basissetexchange.org/ (retrieved March 5, 2020).

(150) Cheng, L.; Gauss, J. Analytic second derivatives for the spin-free exact two-component theory. J. Chem. Phys. 2011, 135, 244104.

(151) Cheng, L.; Gauss, J.; Stanton, J. F. Relativistic coupled-cluster calculations on $\mathrm{XeF}_{6}$ : Delicate interplay between electron-correlation and basis-set effects. J. Chem. Phys. 2015, 142, 224309.

(152) Dirac, P. A. M. Quantum Mechanics of Many-Electron Systems. Proc. Roy. Soc. Lond. A 1929, 123, 714-733. 
(153) Slater, J. C. A Simplification of the Hartree-Fock Method. Phys. Rev. 1951, 81, 385390.

(154) Vosko, S. H.; Wilk, L.; Nusair, M. Accurate spin-dependent electron liquid correlation energies for local spin density calculations: a critical analysis. Can. J. Phys. 1980, $58,1200-1211$.

(155) Keal, T. W.; Tozer, D. J. A semiempirical generalized gradient approximation exchange-correlation functional. J. Chem. Phys. 2004, 121, 5654-5660.

(156) Perdew, J. P. Density-functional approximation for the correlation energy of the inhomogeneous electron gas. Phys. Rev. B 1986, 33, 8822-8824.

(157) Becke, A. D. Density-functional exchange-energy approximation with correct asymptotic behavior. Phys. Rev. A 1988, 38, 3098-3100.

(158) Tao, J.; Perdew, J. P.; Staroverov, V. N.; Scuseria, G. E. Climbing the Density Functional Ladder: Nonempirical Meta-Generalized Gradient Approximation Designed for Molecules and Solids. Phys. Rev. Lett. 2003, 91, 146401.

(159) Perdew, J. P.; Ruzsinszky, A.; Csonka, G. I.; Constantin, L. A.; Sun, J. Workhorse Semilocal Density Functional for Condensed Matter Physics and Quantum Chemistry. Phys. Rev. Lett. 2009, 103, 026403.

(160) Perdew, J. P.; Ruzsinszky, A.; Csonka, G. I.; Constantin, L. A.; Sun, J. Erratum: Workhorse Semilocal Density Functional for Condensed Matter Physics and Quantum Chemistry [Phys. Rev. Lett. 103, 026403 (2009)]. Phys. Rev. Lett. 2011, 106, 179902.

(161) Furness, J. W.; Kaplan, A. D.; Ning, J.; Perdew, J. P.; Sun, J. Accurate and Numerically Efficient $\mathrm{r}^{2} \mathrm{SCAN}$ Meta-Generalized Gradient Approximation. J. Phys. Chem. Lett. 2020, 11, 8208-8215. 
(162) Furness, J. W.; Kaplan, A. D.; Ning, J.; Perdew, J. P.; Sun, J. Correction to "Accurate and Numerically Efficient $\mathrm{r}^{2} \mathrm{SCAN}$ Meta-Generalized Gradient Approximation". J. Phys. Chem. Lett. 2020, 11, 9248-9248.

(163) Lee, C.; Yang, W.; Parr, R. G. Development of the Colle-Salvetti correlation-energy formula into a functional of the electron density. Phys. Rev. B 1988, 37, 785-789.

(164) Becke, A. D. A new mixing of Hartree-Fock and local density-functional theories. $J$. Chem. Phys. 1993, 98, 1372-1377.

(165) Becke, A. D. Density-functional thermochemistry. III. The role of exact exchange. $J$. Chem. Phys. 1993, 98, 5648-5652.

(166) Becke, A. D. Density-functional thermochemistry. V. Systematic optimization of exchange-correlation functionals. J. Phys. Chem. 1997, 107, 8554-8560.

(167) Wilson, P. J.; Bradley, T. J.; Tozer, D. J. Hybrid exchange-correlation functional determined from thermochemical data and ab initio potentials. J. Chem. Phys. 2001, $115,9233-9242$.

(168) Staroverov, V. N.; Scuseria, G. E.; Tao, J.; Perdew, J. P. Comparative assessment of a new nonempirical density functional: Molecules and hydrogen-bonded complexes. $J$. Chem. Phys. 2003, 119, 12129-12137.

(169) Grimme, S. Accurate Calculation of the Heats of Formation for Large Main Group Compounds with Spin-Component Scaled MP2 Methods. J. Phys. Chem. A 2005, 109, 3067-3077.

(170) Yanai, T.; Tew, D. P.; Handy, N. C. A new hybrid exchange-correlation functional using the Coulomb-attenuating method (CAM-B3LYP). Chem. Phys. Lett. 2004, 393, 51-57. 
(171) Jin, Y.; Bartlett, R. J. The QTP family of consistent functionals and potentials in Kohn-Sham density functional theory. J. Chem. Phys. 2016, 145, 034107.

(172) Haiduke, R. L. A.; Bartlett, R. J. Communication: Can excitation energies be obtained from orbital energies in a correlated orbital theory? J. Chem. Phys. 2018, 149, 131101.

(173) Heyd, J.; Scuseria, G. E.; Ernzerhof, M. Hybrid functionals based on a screened Coulomb potential. J. Chem. Phys. 2003, 118, 8207-8215.

(174) Heyd, J.; Scuseria, G. E.; Ernzerhof, M. Erratum: "Hybrid functionals based on a screened Coulomb potential" [J. Chem. Phys. 118, 8207 (2003)]. J. Phys. Chem. 2006, $124,219906$.

(175) Krukau, A. V.; Vydrov, O. A.; Izmaylov, A. F.; Scuseria, G. E. Influence of the exchange screening parameter on the performance of screened hybrid functionals. $J$. Phys. Chem. 2006, 125, 224106.

(176) Vydrov, O. A.; Scuseria, G. E. Assessment of a long-range corrected hybrid functional. J. Chem. Phys. 2006, 125, 234109.

(177) Chai, J.-D.; Head-Gordon, M. Long-range corrected hybrid density functionals with damped atom-atom dispersion corrections. Phys. Chem. Chem. Phys. 2008, 10, 66156620.

(178) Manual of TURBOMOLE V7.5.1 2021, a development of University of Karlsruhe and Forschungszentrum Karlsruhe GmbH, 1989-2007, TURBOMOLE GmbH, since 2007; available from https://www.turbomole.org/turbomole/ turbomole-documentation/ (retrieved August 28, 2021).

(179) Schmitte, J.; Friebel, C.; Weller, F.; Dehnicke, K. Synthese, IR- und EPR-Spektren sowie die Kristallstruktur von $\left(\mathrm{PPh}_{3} \mathrm{Me}_{2}\right)_{2}\left[\mathrm{MoNCl}_{4}\right]$. Z. Anorg. Allg. Chem. 1982, 495, $148-156$. 
(180) Sunil, K. K.; Rogers, M. T. ESR studies of some oxotetrahalo complexes of vanadium(IV) and molybdenum(V). Inorg. Chem. 1981, 20, 3283-3287.

(181) Manoharan, P. T.; Rogers, M. T. Ligand Hyperfine Interactions in Molybdenyl and Chromyl Halide Complexes. J. Chem. Phys. 1968, 49, 5510-5519.

(182) van Kemenade, J. T. C. Ligand Hyperfine Interactions in Oxyhalides of Pentavalent Chromium, Molybdenum and Tungsten. Ph.D. thesis, Technical University Delft, The Netherlands, 1970.

(183) Baldas, J.; Boas, J. F.; Bonnyman, J. Preparation and Properties of Nitridotechnetic (VI) Acid. I. Observation of the ESR-Spectrum of the $\left[\mathrm{TcNF}_{4}\right]^{-}$Anion in Hydrofluoric Acid Solution. Aus. J. Chem. 1989, 42, 639-648.

(184) Baldas, J.; Boas, J. F.; Bonnyman, J.; Williams, G. A. Studies of technetium complexes. Part 6. The preparation, characterisation, and electron spin resonance spectra of salts of tetrachloro- and tetrabromo-nitridotechnetate(VI): crystal structure of tetraphenylarsonium tetrachloronitridotechnetate(VI). J. Chem. Soc., Dalton Trans. 1984, 2395-2400.

(185) Kersting, M.; Friebel, C.; Dehnicke, K.; Krestel, M.; Allmann, R. Synthese, IR- und EPR-Spektren sowie die Kristallstruktur von $\mathrm{HPPh}_{3}\left[\mathrm{WOCl}_{4}\left(\mathrm{OPPh}_{3}\right)\right]$. Z. Anorg. Allg. Chem. 1988, 563, 70-78.

(186) Voigt, A.; Abram, U.; Kirmse, R. The existence of $\left[\mathrm{ReNF}_{4}\right]^{-}$- an EPR study. Inorg. Chem. Commun. 1998, 1, 141-142.

(187) Voigt, A.; Abram, U.; Böttcher, R.; Richter, U.; Reinhold, J.; Kirmse, R. Q-Band single-crystal EPR study and molecular orbital calculations of $\left[\left(\mathrm{C}_{6} \mathrm{H}_{5}\right)_{4} \mathrm{As}\right]\left[\mathrm{Re}^{\mathrm{VI}} \mathrm{NCl}_{4} / \mathrm{Re}^{\mathrm{V}} \mathrm{OCl}_{4}\right]$. Chem. Phys. 2000, 253, 171-181. 
(188) Abram, U.; Braun, M.; Abram, S.; Kirmse, R.; Voigt, A. [NBu 4$]\left[\mathrm{ReNCl}_{4}\right]$ : Facile synthesis, structure, electron paramagnetic resonance spectroscopy and reactions. $J$. Chem. Soc., Dalton Trans. 1998, 231-238.

(189) Borisova, L. V.; Ermakov, A. N.; Plastinina, Y. I.; Prasolova, O. D.; Marov, I. N. Determination of rhenium based on the formation of rhenium(VI) oxide halide complexes by spectrophotometry and electron spin resonance spectroscopy. Analyst 1982, 10\%, $500-504$.

(190) Holloway, J. H.; Hope, E. G.; Raynor, J. B.; Townson, P. T. Magnetic resonance studies on osmium pentafluoride oxide. J. Chem. Soc., Dalton Trans. 1992, 1131-1134.

(191) Dyall, K. G.; Gomes, A. S. P. Revised relativistic basis sets for the 5d elements Hf-Hg. Theor. Chem. Acc. 2009, 125, 97-100.

(192) Grimme, S.; Antony, J.; Ehrlich, S.; Krieg, H. A consistent and accurate ab initio parametrization of density functional dispersion correction (DFT-D) for the 94 elements H-Pu. J. Chem. Phys. 2010, 132, 154104.

(193) Gould, C. A.; McClain, K. R.; Yu, J. M.; Groshens, T. J.; Furche, F.; Harvey, B. G.; Long, J. R. Synthesis and Magnetism of Neutral, Linear Metallocene Complexes of Terbium(II) and Dysprosium(II). J. Am. Chem. Soc. 2019, 141, 12967-12973.

(194) Yu, J. M.; Furche, F. Theoretical Study of Divalent Bis(Pentaisopropylcyclopentadienyl) Actinocenes. Inorg. Chem. 2019, 58, 1600416010.

(195) Ryan, A. J.; Darago, L. E.; Balasubramani, S. G.; Chen, G. P.; Ziller, J. W.; Furche, F.; Long, J. R.; Evans, W. J. Synthesis, structure, and magnetism of tris(amide $)\left[\operatorname{Ln}\left\{\mathrm{N}\left(\mathrm{SiMe}_{3}\right)_{2}\right\}_{3}\right]^{1-}$ complexes of the non-traditional +2 lanthanide ions. Chem.: Eur. J. 2018, 24, 7702-7709. 
(196) MacDonald, M. R.; Bates, J. E.; Ziller, J. W.; Furche, F.; Evans, W. J. Completing the series of +2 ions for the lanthanide elements: synthesis of molecular complexes of $\mathrm{Pr}^{2+}, \mathrm{Gd}^{2+}, \mathrm{Tb}^{2+}$, and $\mathrm{Lu}^{2+}$. J. Am. Chem. Soc. 2013, 135, 9857-9868.

(197) Fieser, M. E.; MacDonald, M. R.; Krull, B. T.; Bates, J. E.; Ziller, J. W.; Furche, F.; Evans, W. J. Structural, spectroscopic, and theoretical comparison of traditional vs recently discovered $\mathrm{Ln}^{2+}$ ions in the $[\mathrm{K}(2.2 .2$ - cryptand $)]\left[\left(\mathrm{C}_{5} \mathrm{H}_{4} \mathrm{SiMe}_{3}\right)_{3} \mathrm{Ln}\right]$ complexes: the variable nature of $\mathrm{Dy}^{2+}$ and $\mathrm{Nd}^{2+}$. J. Am. Chem. Soc. 2014, 137, 369-382.

(198) Windorff, C. J.; Chen, G. P.; Cross, J. N.; Evans, W. J.; Furche, F.; Gaunt, A. J.; Janicke, M. T.; Kozimor, S. A.; Scott, B. L. Identification of the formal +2 oxidation state of plutonium: synthesis and characterization of $\left\{\mathrm{Pu}^{\mathrm{II}}\left[\mathrm{C}_{5} \mathrm{H}_{3}\left(\mathrm{SiMe}_{3}\right)_{2}\right]_{3}\right\}^{-} . J . A m$. Chem. Soc. 2017, 139, 3970-3973.

(199) Nava, P.; Sierka, M.; Ahlrichs, R. Density functional study of palladium clusters. Phys. Chem. Chem. Phys. 2003, 5, 3372-3381.

(200) Branzoli, F.; Carretta, P.; Filibian, M.; Zoppellaro, G.; Graf, M. J.; GalanMascaros, J. R.; Fuhr, O.; Brink, S.; Ruben, M. Spin Dynamics in the Negatively Charged Terbium (III) Bis-phthalocyaninato Complex. J. Am. Chem. Soc. 2009, 131, 4387-4396.

(201) Sikkema, J.; Visscher, L.; Saue, T.; Iliaš, M. The molecular mean-field approach for correlated relativistic calculations. J. Chem. Phys. 2009, 131, 124116.

(202) Chesnut, D. B.; Moore, K. D. Locally dense basis sets for chemical shift calculations. J. Comput. Chem. 1989, 10, 648-659.

(203) Chesnut, D.; Rusiloski, B.; Moore, K.; Egolf, D. Use of locally dense basis sets for nuclear magnetic resonance shielding calculations. J. Comput. Chem. 1993, 14, 13641375. 
(204) Malkin, E.; Malkin, I.; Malkina, O. L.; Malkin, V. G.; Kaupp, M. Scalar relativistic calculations of hyperfine coupling tensors using the Douglas-Kroll-Hess method with a finite-size nucleus model. Phys. Chem. Chem. Phys. 2006, 8, 4079-4085.

(205) Autschbach, J. Magnitude of Finite-Nucleus-Size Effects in Relativistic Density Functional Computations of Indirect NMR Nuclear Spin-Spin Coupling Constants. ChemPhysChem 2009, 10, 2274-2283.

(206) Lehtola, S.; Dimitrova, M.; Fliegl, H.; Sundholm, D. Benchmarking Magnetizabilities with Recent Density Functionals. J. Chem. Theory Comput. 2021, 17, 1457-1468.

(207) Lehtola, S.; Dimitrova, M.; Fliegl, H.; Sundholm, D. Correction to "Benchmarking Magnetizabilities with Recent Density Functionals". J. Chem. Theory Comput. 2021, $17,1457-1468$.

(208) Holzer, C.; Franzke, Y. J.; Kehry, M. Assessing the Accuracy of Local Hybrid Density Functional Approximations for Molecular Response Properties. J. Chem. Theory Comput. 2021, 17, 2928-2947.

(209) Teale, A. M.; Lutnæs, O. B.; Helgaker, T.; Tozer, D. J.; Gauss, J. Benchmarking density-functional theory calculations of NMR shielding constants and spin-rotation constants using accurate coupled-cluster calculations. J. Chem. Phys. 2013, 138, 024111.

(210) Hedegård, E. D.; Kongsted, J.; Sauer, S. P. A. Validating and Analyzing EPR Hyperfine Coupling Constants with Density Functional Theory. J. Chem. Theory Comput. 2013, 9, 2380-2388.

(211) Flaig, D.; Maurer, M.; Hanni, M.; Braunger, K.; Kick, L.; Thubauville, M.; Ochsenfeld, C. Benchmarking Hydrogen and Carbon NMR Chemical Shifts at HF, DFT, and MP2 Levels. J. Chem. Theory Comput. 2014, 10, 572-578. 
(212) Stoychev, G. L.; Auer, A. A.; Izsák, R.; Neese, F. Self-Consistent Field Calculation of Nuclear Magnetic Resonance Chemical Shielding Constants Using Gauge-Including Atomic Orbitals and Approximate Two-Electron Integrals. J. Chem. Theory Comput. 2018, 14, 619-637.

(213) Reiter, K.; Mack, F.; Weigend, F. Calculation of Magnetic Shielding Constants with meta-GGA Functionals Employing the Multipole-Accelerated Resolution of the Identity: Implementation and Assessment of Accuracy and Efficiency. J. Chem.Theory Comput. 2018, 14, 191-197.

(214) Lutnæs, O. B.; Teale, A. M.; Helgaker, T.; Tozer, D. J.; Ruud, K.; Gauss, J. Benchmarking density-functional-theory calculations of rotational g tensors and magnetizabilities using accurate coupled-cluster calculations. J. Chem. Phys. 2009, 131, 144104.

(215) Eichkorn, K.; Weigend, F.; Treutler, O.; Ahlrichs, R. Auxiliary basis sets for main row atoms and transition metals and their use to approximate Coulomb potentials. Theor. Chem. Acc. 1997, 97, 119-124.

(216) Weigend, F. Accurate Coulomb-fitting basis sets for H to Rn. Phys. Chem. Chem. Phys. 2006, 8, 1057-1065.

(217) Weigend, F.; Häser, M.; Patzelt, H.; Ahlrichs, R. RI-MP2: optimized auxiliary basis sets and demonstration of efficiency. Chem. Phys. Lett. 1998, 294, 143-152.

(218) Weigend, F.; Köhn, A.; Hättig, C. Efficient use of the correlation consistent basis sets in resolution of the identity MP2 calculations. J. Chem. Phys. 2002, 116, 3175-3183.

(219) Hättig, C. Optimization of auxiliary basis sets for RI-MP2 and RI-CC2 calculations: Core-valence and quintuple-zeta basis sets for $\mathrm{H}$ to Ar and QZVPP basis sets for Li to Kr. Phys. Chem. Chem. Phys. 2005, 7, 59-66. 
(220) Eshuis, H.; Yarkony, J.; Furche, F. Fast computation of molecular random phase approximation correlation energies using resolution of the identity and imaginary frequency integration. J. Chem. Phys. 2010, 132, 234114.

(221) Alonso, P.; Alcalá, R.; Usón, R.; Forniés, J. EPR study of mononuclear Pt(III) organometallic complexes. J. Phys. Chem. Solids 1991, 52, 975-978.

(222) Jaramillo, J.; Scuseria, G. E.; Ernzerhof, M. Local hybrid functionals. J. Chem. Phys. 2003, 118, 1068-1073.

(223) Wodyński, A.; Kaupp, M. Noncollinear Relativistic Two-Component X2C Calculations of Hyperfine Couplings Using Local Hybrid Functionals. Importance of the HighDensity Coordinate Scaling Limit. J. Chem. Theory Comput. 2020, 16, 314-325.

(224) Johnson, E. R. Local-hybrid functional based on the correlation length. J. Chem. Phys. 2014, 141, 124120.

(225) Perdew, J. P.; Staroverov, V. N.; Tao, J.; Scuseria, G. E. Density functional with full exact exchange, balanced nonlocality of correlation, and constraint satisfaction. Phys. Rev. A 2008, 78, 052513.

(226) Ishikawa, N.; Sugita, M.; Wernsdorfer, W. Quantum Tunneling of Magnetization in Lanthanide Single-Molecule Magnets: Bis(phthalocyaninato)terbium and Bis(phthalocyaninato)dysprosium Anions. Angew. Chem. Int. Ed. 2005, 44, 29312935.

(227) Taran, G.; Bonet, E.; Wernsdorfer, W. The role of the quadrupolar interaction in the tunneling dynamics of lanthanide molecular magnets. J. Appl. Phys. 2019, 125, 142903.

(228) Repiský, M.; Komorovský, S.; Malkin, E.; Malkina, O. L.; Malkin, V. G. Relativistic 
four-component calculations of electronic g-tensors in the matrix Dirac-Kohn-Sham framework. Chem. Phys. Lett. 2010, 488, 94-97.

(229) London, F. Théorie quantique des courants interatomiques dans les combinaisons aromatiques. J. Phys. Radium 1937, 8, 397-409.

(230) Ditchfield, R. Self-consistent perturbation theory of diamagnetism. Mol. Phys. 1974, 27, 789-807. 